Research Paper

\title{
RNA-sequencing investigation identifies an effective risk score generated by three novel IncRNAs for the survival of papillary thyroid cancer patients
}

\author{
Yi-Huan Luo ${ }^{1, *}$, Liang Liang ${ }^{2, *}$, Rong-Quan $\mathrm{He}^{3}$, Dong-Yue Wen ${ }^{4}$, Guo-Fei Deng ${ }^{1}$, \\ Hong Yang ${ }^{4}$, Yun $\mathrm{He}^{4}$, Wei Ma ${ }^{5}$, Xiao-Yong Cai ${ }^{2}$, Jun-Qiang Chen ${ }^{1}$ and Gang Chen ${ }^{5}$ \\ ${ }^{1}$ Department of Gastrointestinal Surgery, First Affiliated Hospital of Guangxi Medical University, Nanning, Guangxi Zhuang \\ Autonomous Region, P.R. China \\ ${ }^{2}$ Department of General Surgery, First Affiliated Hospital of Guangxi Medical University (West Branch), Nanning, Guangxi \\ Zhuang Autonomous Region, P.R. China \\ ${ }^{3}$ Department of Medical Oncology, First Affiliated Hospital of Guangxi Medical University, Nanning, Guangxi Zhuang \\ Autonomous Region, P.R. China \\ ${ }^{4}$ Department of Ultrasonography, First Affiliated Hospital of Guangxi Medical University, Nanning, Guangxi Zhuang \\ Autonomous Region, P.R. China \\ ${ }^{5}$ Department of Pathology, First Affiliated Hospital of Guangxi Medical University, Nanning, Guangxi Zhuang Autonomous \\ Region, P.R. China \\ "These authors contributed equally to this work
}

Correspondence to: Jun-Qiang Chen, email: nnhans@163.com

Gang Chen, email: chen_gang_triones@163.com

Keywords: papillary thyroid cancer, IncRNA, prognosis, AC079630.2, CRNDE

Received: January 30, 2017 Accepted: May 15, $2017 \quad$ Published: May 26, 2017

Copyright: Luo et al. This is an open-access article distributed under the terms of the Creative Commons Attribution License 3.0 (CC BY

3.0), which permits unrestricted use, distribution, and reproduction in any medium, provided the original author and source are credited.

\section{ABSTRACT}

Scholars are striving to apply molecular biology involving long non-coding RNA (IncRNA) in the prognostication of papillary thyroid cancer (PTC). However, the clinical role of IncRNAs in the prognostic setting of PTC is still unclear. Herein, a comprehensive inquiry was performed to screen IncRNA expression profiling with 507 PTC patients from The Cancer Genome Atlas RNA-sequencing datasets. A total of 734 IncRNAs were detected to be aberrantly expressed, among which three novel IncRNAs including AC079630.2, CRNDE and CTD-2171N6.1 were markedly related to the progression and survival of PTC. Furthermore, the aberrant expression of these IncRNAs could be verified by other cohorts from gene expression omnibus (GEO) as detected by microarrays. Next, we established a three-IncRNA signature and divided the PTC patients into two subgroups of high- and low-risk. Interestingly, patients with high-risk tended to gain obviously poorer outcome. Most importantly, this threeIncRNA signature was an independent biomarker to predict the patient survival of PTC. The accurate molecular roles of these three IncRNAs remains unclarified and warrants further characterization, but our current data propose that they might play pivotal roles in PTC tumorigenesis and more importantly, these novel IncRNAs are closely related to patients' survival. These discoveries will have far-reaching consequences with respect to molecular prediction of PTC. 


\section{INTRODUCTION}

Thyroid cancer is the most widespread class of endocrine malignancies. The occurrence of thyroid cancer has been gradually growing over the last 30 years $[1,2]$. It was estimated that 64,300 new cases of thyroid cancer occurred in the United States in 2016, and 1,980 of them deceased (https://www.cancer.org/). Women are more prone to be affected by thyroid cancer than men. People between the ages of 25 and 65 are the high-risk group to suffer thyroid cancer $[3,4]$. There are four subtypes of thyroid cancer according to the histopathological examination, including papillary, follicular, medullary and anaplastic thyroid cancer. Generally, thyroid cancer is divided into two classes: well-differentiated (papillary or follicular) and poorly-differentiated (medullary or anaplastic), which also lead to various clinical outcomes. For most of thyroid cancer, the therapeutic options including operation, radioactive iodine ablation, and thyroid stimulating hormone (TSH) suppressive therapy provide an overall survival (OS) rate of $97.7 \%$ at 5 years. Nonetheless, locoregional recurrence takes place in up to $20 \%$ of thyroid cancer patients. More seriously, distant metastases will occur in almost $10 \%$ of thyroid cancer patients in 10years [5-7].

The molecular biology has been greatly improved in the prognostication of papillary thyroid cancer (PTC). Several molecular biomarkers have been commended for the risk stratification for PTC, along with traditional clinicopathological parameters. The typical examples are $\mathrm{BRAF}^{\mathrm{V} 600 \mathrm{E}}$ and telomerase reverse transcriptase (TERT) [8-14]. Nonetheless, the clinical characteristics of molecular characterization in the prognostic setting of PTC still remain unclarified. A deeper understanding of the drivers and molecular mechanisms behind PTC tumorigenesis, as well as documentation of candidate prognostic markers, is essential to put forward better diagnostic and therapeutic strategies for PTC [15-17].

Long noncoding RNAs (lncRNAs) have been proved to play essential roles in cancer biology, including PTC. The detection of transcriptome profile of PTC is an ideal option to screen and discover new lncRNA candidates [18]. By far, several groups performed genomewide investigation into lncRNAs expression profile in PTC using microarray $[19,20]$ or RNA sequencing [21-23]. However, these profilings were all based on a small number of cases and the results were varying with each other. The cancer genome atlas (TCGA) has made great efforts to unveil the molecular events of various cancers, including the role of lncRNAs. Ma et al. [24] mined the data from TCGA dataset and they reported 220 lncRNAs with altered expression from the annotated 2773 lncRNAs which were approved by the HUGO gene nomenclature committee via cBioPortal. They further reported that FAM41C, CTBP1-AS2, LINC00271, HAR1A, LINC00310 and HAS2-AS1 were related to recurrence of PTC patients. More importantly, only one IncRNA, LINC00271 was identified to be an independent risk factor for the progress and recurrence of PTC in multivariate analyses. In the current study, the genomewide lncRNA expression data from the high-throughput RNA-sequencing dataset was also achieved from TCGA; however, 7589 lncRNAs were annotated by Ensembl genome browser (www.ensembl.org/). More interestingly, we used DEseq R package to screen the differentially expressed lncRNAs and the results differed from Ma et al. [24]. We further selected those significant prognostic lncRNAs to form a signature. The three-lncRNA signature can be an effective independent indicator to predict the progression and survival of PTC.

\section{RESULTS}

\section{Selection of differentially expressed IncRNAs}

Initially, detailed information of 507 PTC patients and 59 normal thyroid tissues was obtained from TCGA dataset. A sum of 734 lncRNAs were selected as differentially expressed lncRNA in PTC compared to normal thyroid tissues, of which 309 lncRNAs were down-regulated and 425 lncRNAs were up-regulated. The overview of aberrant expression lncRNAs was shown in Figure 1A, 1B.

\section{Specific-IncRNAs correlated with clinical parameters and overall survival (OS)}

With respect to the correlation between lncRNAs and OS, 33 lncRNAs were assessed to be significantly associated with OS of PTC patients by univariate analysis (Table 1). In the light of multivariate analysis, a total of 3 lncRNAs (AC079630.2, CRNDE and CTD-2171N6.1) were remarkably correlated with OS and these lncRNAs were subjected to further analysis (Table 2). We also investigated the association between these lncRNAs and disease-free survival (DFS), but limited predicted significance was observed (Figure 2). Fifty-three patients were excluded for the reason that they lacked specific IncRNAs (AC079630.2, CRNDE or CTD-2171N6.1) expression data. Eventually, 449 patients were included for further analysis in prognosis model. The available data of clinicopathological parameters of the involved patients were summarized in Table 3. Spearman rank correlation was performed to determine the association between these three lncRNAs and clinicopathological variables (Table 4). The results indicated that AC079630.2 was prominently related to tumor size $(P=0.005)$, CRNDE was remarkably related to gender $(P=0.016)$ and CTD-2171N6.1 was significantly related to tumor subtypes $(P=0.001)$. Additionally, the three lncRNAs-based risk score was observed to be notably related to age $(P=0.004)$ and tumor subtypes $(P=0.013)$. 


\section{Specific IncRNAs featured with diagnostic significance}

The expression of these three lncRNAs was greatly higher in PTC tissues than that in normal thyroid tissues based on the data from TCGA. Receiver operating characteristic (ROC) curve was conducted to verify the diagnostic value of the three novel lncRNAs in PTC patients. The results of ROC analysis indicated that AC079630.2 exhibited high diagnostic ability to distinguish normal tissue and PTC tissue. The areas under curves (AUC) of these three lncRNAs (AC079630.2, CRNDE and CTD-2171N6.1) were 0.940 (95\% CI: $0.917-$
0.958), 0.677 (95\% CI: 0.636-0.715), 0.664 (95\% CI: 0.623-0.703), respectively (Figure 3). Moreover, a total of 10 microarrays from GEO were achieved in our study to heighten our finding from TCGA, of which two microarrays contained the data of AC079630.2, 10 for CRNDE, and two for CTD-2171N6.1. The expression level of these three lncRNAs was shown in Table 5. The expression level of AC079630.2 in PTC was evidently higher than that in normal thyroid tissues as shown in both GSE83520 and GSE64912. The AUCs were 1.000 (95\% CI: $0.858-1.000)$ and 0.986 (95\% CI: 0.821-1.000), which demonstrated favorable diagnostic value of AC079630.2 in PTC (Figure 4). Among the 10 microarray data containing

A

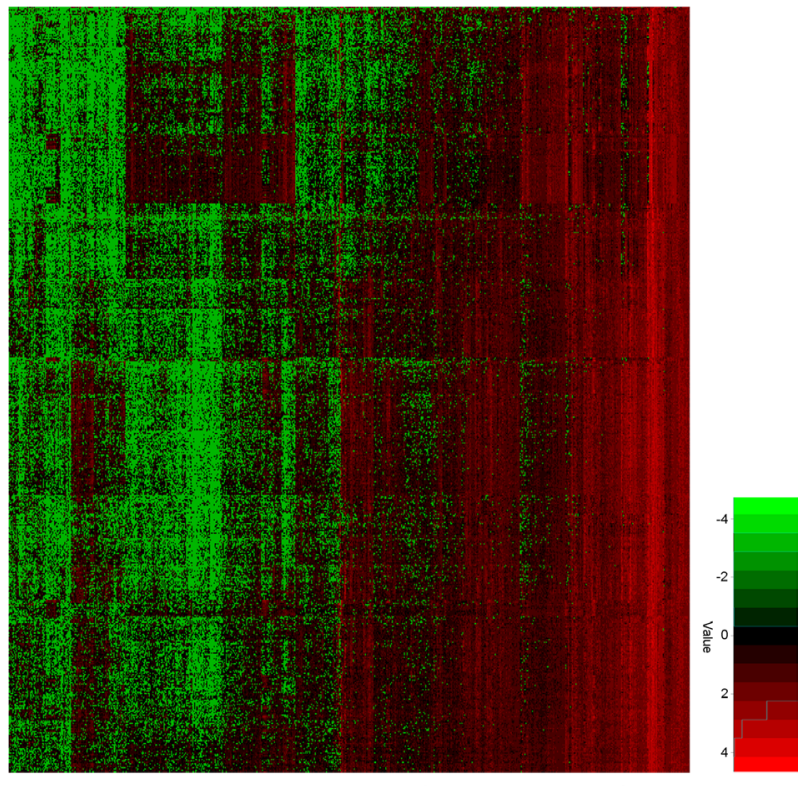

B

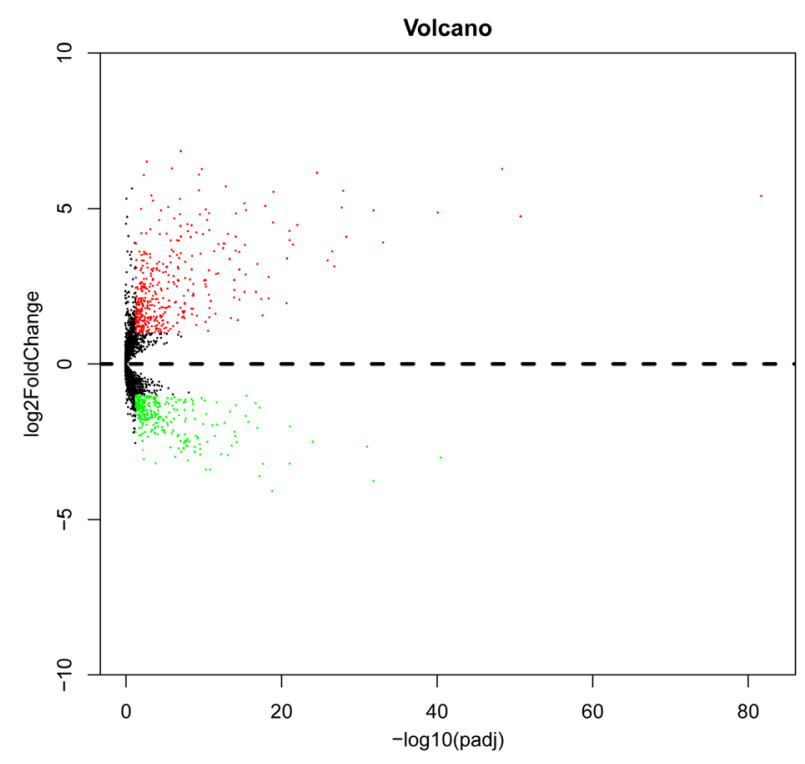

Figure 1: The expression level of upregulated and downregulated IncRNAs in PTC tissues. (A) heat map; (B) volcano map. 
Table 1: Specific IncRNAs significantly associated with OS by univariate analysis (TCGA dataset, $N=502)$

\begin{tabular}{|c|c|c|}
\hline LncRNAs name & HR & $P$ \\
\hline AC079630.2 & 0.818 & 0.002 \\
\hline CRNDE & 1.796 & 0.003 \\
\hline CTD-2171N6.1 & 1.526 & 0.009 \\
\hline RP3-449M8.9 & 0.647 & 0.002 \\
\hline KB-208E9.1 & 0.670 & 0.004 \\
\hline RP11-320N7.2 & 0.707 & 0.004 \\
\hline RP11-476D10.1 & 0.827 & 0.005 \\
\hline LINC00900 & 0.613 & 0.005 \\
\hline RP11-1134I14.8 & 0.685 & 0.005 \\
\hline RP4-665J23.1 & 1.643 & 0.009 \\
\hline KB-1732A1.1 & 1.946 & 0.011 \\
\hline RP11-20J15.3 & 0.815 & 0.011 \\
\hline RPL34-AS1 & 1.898 & 0.012 \\
\hline CITF22-49E9.3 & 1.658 & 0.014 \\
\hline RP11-359G22.2 & 0.727 & 0.018 \\
\hline RP11-539L10.2 & 0.726 & 0.022 \\
\hline AP001258.4 & 0.591 & 0.024 \\
\hline MIR100HG & 1.539 & 0.029 \\
\hline RP11-324E6.10 & 0.626 & 0.030 \\
\hline RP11-193M21.1 & 1.601 & 0.030 \\
\hline RP11-120D5.1 & 1.822 & 0.030 \\
\hline CTC-523E23.1 & 0.646 & 0.030 \\
\hline RP11-539G18.3 & 1.549 & 0.030 \\
\hline RP11-879F14.2 & 0.678 & 0.031 \\
\hline RP11-221N13.3 & 0.827 & 0.032 \\
\hline RP11-295M3.4 & 0.630 & 0.033 \\
\hline AC104655.3 & 0.765 & 0.034 \\
\hline RP11-299H21.1 & 1.589 & 0.044 \\
\hline LINC01314 & 0.829 & 0.045 \\
\hline RP11-42O15.3 & 1.532 & 0.046 \\
\hline CH17-373J23.1 & 1.602 & 0.046 \\
\hline RP3-449M8.6 & 0.767 & 0.047 \\
\hline LINC00284 & 0.801 & 0.049 \\
\hline
\end{tabular}

HR: hazard ratio.

CRNDE expression, four indicated that CRNDE possessed a certain degree of diagnostic value for PTC, including GSE35570: 0.735 (95\%CI: 0.645-0.813), GSE3678: 0.918 (95\% CI: 0.648-0.997), GSE60542: 0.734 (95\% CI: 0.6080.838), and GSE83520: 0.728 (95\% CI: 0.472-0.907) (Figure 5A-5J and Figure 6). But no significant distinction of CTD-2171N6.1 expression between PTC and normal thyroid was observed in GEO datasets (Figure 5K, 5L). In addition, we conducted meta-analyses of AC079630.2

and CRNDE to explore their diagnostic significances. The results indicated that the pooled AUCs were 0.975 and 0.809 for these two lncRNAs respectively (Figure 7).

\section{Prognostic significance of clinicopathological} parameters and the three-IncRNA signature in PTC

For each patient, a specific risk score was calculated with 3 recruited lncRNAs (AC079630.2, CRNDE and 
Table 2: The three IncRNAs significantly correlated with OS by univariate and multivariate analysis in PTC patients (TCGA dataset, $N=502$ )

\begin{tabular}{|c|c|c|c|c|c|c|c|}
\hline \multirow{2}{*}{$\begin{array}{l}\text { LncRNAs } \\
\text { name }\end{array}$} & \multirow[t]{2}{*}{ Ensemble ID } & \multirow[t]{2}{*}{ Location } & \multicolumn{2}{|c|}{$\begin{array}{l}\text { Univariate } \\
\text { analysis }\end{array}$} & \multicolumn{3}{|c|}{$\begin{array}{l}\text { Multivariate } \\
\text { analysis }\end{array}$} \\
\hline & & & HR & $P$ & $\beta$ & HR (95\% CI) & $P$ \\
\hline AC079630.2 & ENSG00000223914 & $\begin{array}{l}\text { Chromosome 12: } \\
40,156,239-40,167,707\end{array}$ & 0.818 & 0.002 & -0.344 & $0.709(0.548-0.918)$ & 0.009 \\
\hline CRNDE & ENSG00000245694 & $\begin{array}{l}\text { Chromosome 16: } \\
54,845,189-54,929,189\end{array}$ & 1.796 & 0.003 & 0.787 & $2.196(1.044-4.622)$ & 0.038 \\
\hline $\begin{array}{l}\text { CTD- } \\
\text { 2171N6.1/ } \\
\text { LINC01929 }\end{array}$ & ENSG00000267013 & $\begin{array}{l}\text { Chromosome 18: } \\
55,105,904-55,124,306\end{array}$ & 1.526 & 0.009 & 0.885 & $2.422(1.357-4.321)$ & 0.003 \\
\hline
\end{tabular}

CTD-2171N6.1). The risk score $=-0.344 \times$ level of AC079630.2 + $0.787 \times$ level of CRNDE $+0.885 \times$ level of CTD-2171N6.1. The coefficients above were obtained from the $\beta$ value in Table 2 . Then, all patients were divided into low risk group $(N=364)$ and high risk group $(N=85)$ by optimal cut off value. The expression value of the 3 IncRNAs was performed in the heatmap (Figure 8A). The risky lncRNAs (CRNDE, CTD-2171N6.1) feature high expression in high risk group, while the protective lncRNA (AC079630.2) expresses decreased level in high risk group. The Kaplan-Meier survival curve indicated that patients with high risk score were significantly associated with poor prognosis in PTC (Figure 8B). Considering the correlation of risk score with clinicopathological features, the results of Spearman rank correlation revealed that 3 lncRNAs-based risk score was notably related to age $(P=0.004)$ and tumor subtypes $(P=0.013)$, which indicating that elder patients had a higher risk score.

The univariate and multivariate Cox proportional hazards regression tests were carried out to explore the prognostic value of clinical factors and risk score. For univariate analysis, we found that age, history of other tumor, pathological stage, $\mathrm{M}$ stage and risk score were remarkably associated with worse survival status
$(P<0.05)$. After adjusting concomitant variables, the results of multivariate analysis indicated that age $(\mathrm{HR}=1.148,95 \% \mathrm{CI}: 1.070-1.232, P<0.001)$ and risk score were independent prognostic indicators in PTC $(\mathrm{HR}=5.650,95 \%$ CI: 1.188-26.875, $P=0.030)$ (Table 6). In addition, Kaplan-Meier survival curve and log-rank method were further used for the subgroup of clinical features which were significantly associated with OS in univariate analysis. For younger patients, survival analysis was not conducted as all patients were censored. The OS of patients with high risk score were remarkably lower than those with low risk score in elder patients (Figure 9A, $P<0.001)$. When patients were divided into two groups of with and without the history of other tumors, the risk score model exhibited significant prognostic power in both two subgroups (Figure 9B, $P=0.007$; Figure 9C, $P=0.001$ ). Similarly, survival analysis was performed for subgroups of early stage (I-II) and advanced stage (III-IV). The results indicated that the OS time of high risk score group were significant shorter than that of low risk score in patients with advanced stage (Figure 9E, $P<0.001$ ). However, no clear difference was observed in patients with early stage (Figure 9D, $P=0.326$ ). Furthermore, significant difference of OS time was detected between
A

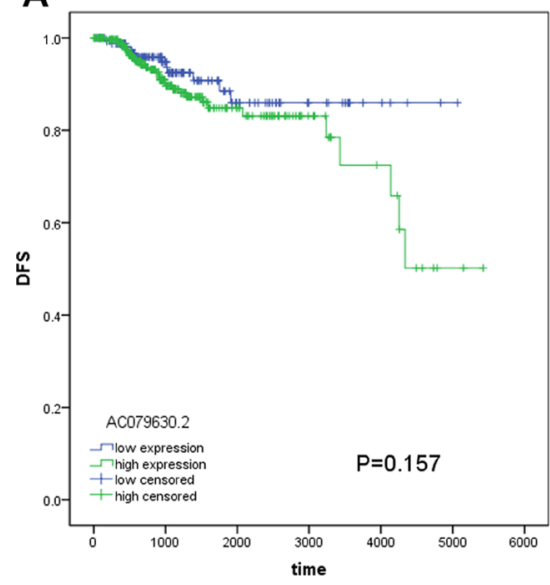

B

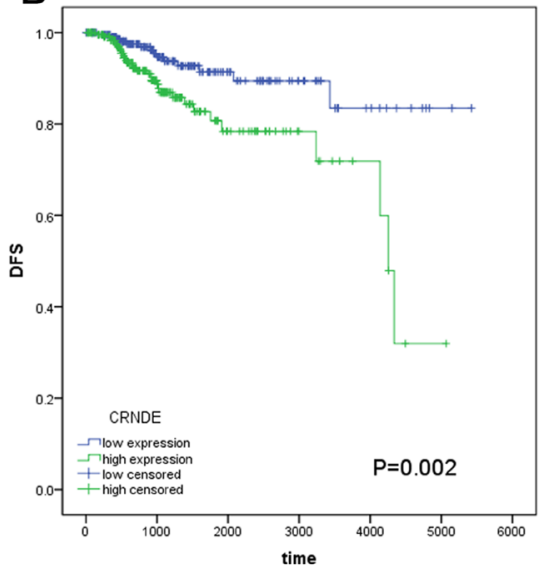

C

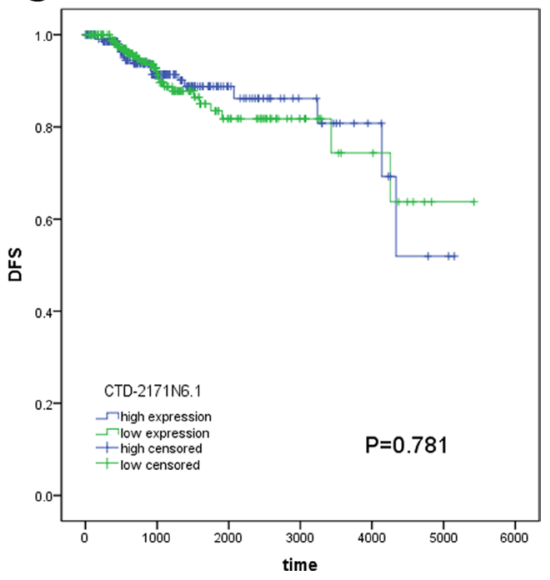

Figure 2: The prognostic significance of these IncRNAs for DFS. (A) AC079630.2; (B) CRNDE; (C) CTD-2171N6.1. 
Table 3: The characteristics of included PTC patients (TCGA dataset, $N=449$ )

\begin{tabular}{|c|c|c|}
\hline \multicolumn{2}{|l|}{ Pathological parameters } & \multirow{2}{*}{$\frac{\text { No. of patients } \boldsymbol{N}(\mathbf{\%})}{198(45.3)}$} \\
\hline Age & $<45$ & \\
\hline \multirow{3}{*}{ Gender } & $\geq 45$ & $239(54.7)$ \\
\hline & Male & $122(27.9)$ \\
\hline & Female & $315(72.1)$ \\
\hline \multirow[t]{4}{*}{ Tumor subtypes } & Classical/Usual & $310(70.9)$ \\
\hline & Tall cell & $33(7.6)$ \\
\hline & Follicular & $89(20.4)$ \\
\hline & Others & $5(1.1)$ \\
\hline \multirow[t]{2}{*}{ History of other tumor } & No & $406(94.2)$ \\
\hline & Yes & $25(5.8)$ \\
\hline \multirow[t]{2}{*}{ Pathological stage } & I-II & $285(65.5)$ \\
\hline & III-IV & $150(34.5)$ \\
\hline \multirow[t]{2}{*}{ T stage } & $\mathrm{T} 1-\mathrm{T} 2$ & $264(60.4)$ \\
\hline & T3-T4 & $173(39.6)$ \\
\hline \multirow[t]{2}{*}{ N stage } & $\mathrm{Nx}-\mathrm{N} 0$ & $236(54.0)$ \\
\hline & N1 & $201(46.0)$ \\
\hline \multirow[t]{2}{*}{ M stage } & M0 & $428(98.2)$ \\
\hline & M1 & $8(1.8)$ \\
\hline \multirow[t]{2}{*}{ Focus types } & Unifocal & $228(53.3)$ \\
\hline & Multifocal & $200(46.7)$ \\
\hline \multirow[t]{2}{*}{ Extrathyroidal extension } & No & $280(66.2)$ \\
\hline & Yes & $143(33.8)$ \\
\hline \multirow{2}{*}{$\mathrm{BRAF}^{\mathrm{V} 600 \mathrm{E}}$} & Mutation type & $212(48.5)$ \\
\hline & Wild type & $225(51.5)$ \\
\hline
\end{tabular}

Table 4: The correlation of three IncRNAs and risk score with clinicopathological parameters (TCGA dataset, $N=449$ )

\begin{tabular}{lcccc}
\hline Clinicopathological parameters & AC079630.2 $(\boldsymbol{P})$ & CRNDE $(\boldsymbol{P})$ & CTD-2171N6.1 $(\boldsymbol{P})$ & Risk score $(\boldsymbol{P})$ \\
\hline Age & 0.122 & 0.115 & 0.092 & $\mathbf{0 . 0 0 4}$ \\
Gender & 0.602 & $\mathbf{0 . 0 1 6}$ & 0.077 & 0.963 \\
Tumor subtypes & 0.508 & 0.226 & $\mathbf{0 . 0 0 1}$ & $\mathbf{0 . 0 1 3}$ \\
History of other tumor & 0.71 & 0.265 & 0.237 & 0.226 \\
Pathological stage & 0.598 & 0.074 & 0.523 & 0.319 \\
T stage & 0.612 & 0.346 & 0.342 & 0.303 \\
N stage & 0.057 & 0.698 & 0.062 & 0.308 \\
M stage & 0.805 & 0.209 & 0.137 & 0.173 \\
Focus types & 0.261 & 0.922 & 0.393 & 0.971 \\
Extrathyroidal extension & 0.666 & 0.251 & 0.264 & 0.872 \\
BRAFV600 & 0.982 & 0.105 & $\mathbf{0 . 0 4 5}$ & $\mathbf{0 . 0 0 6}$ \\
\hline$P . P-$ Vale
\end{tabular}

$P: P$ - values of Spearman rank correlation. 
high risk score group and low risk score group in patients with M0 stage (Figure 9F, $P<0.001$ ) but not M1 stage (Figure 9G, $P=0.156$ ). In subtypes of tall cell PTC, survival analysis failed to be performed because of lacking ending events. Interestingly, the OS time of classical/usual PTC patients with low risk score was significantly longer than those with high risk score (Figure 9H, $P<0.001$ ) and there was no statistical significance among follicular PTC patients (Figure 9I, $P=0.0543$ ). Unfortunately, no survival data of AC079630.2, CRNDE or CTD-2171N6.1 in PTC could be obtained from GEO datasets.
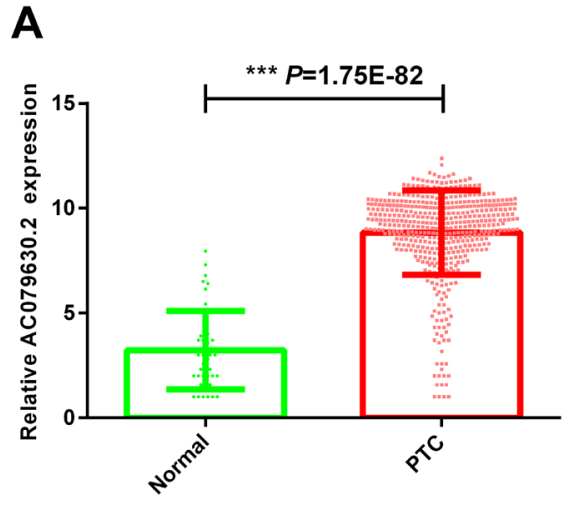

C
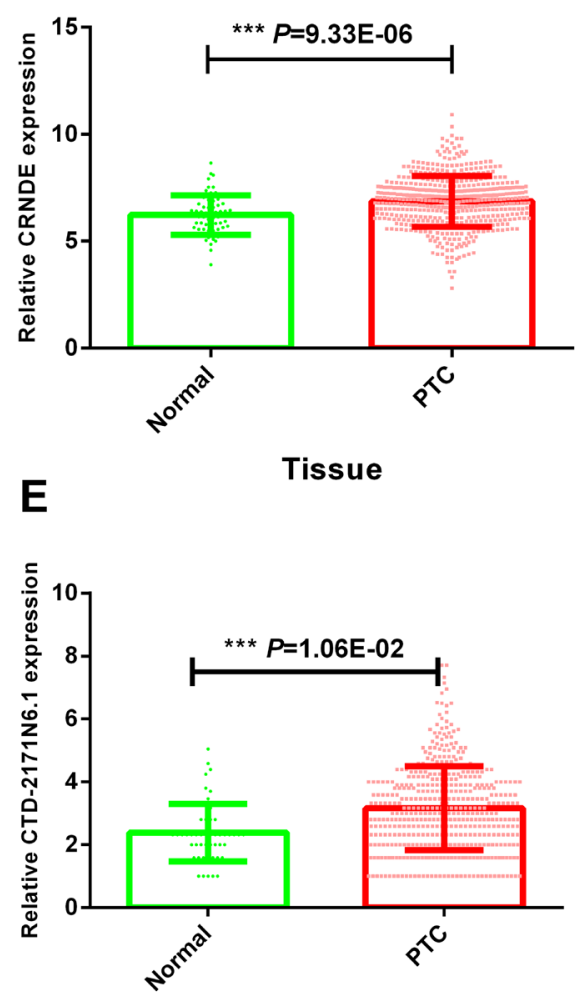

Tissue

\section{Functional and protein-protein interaction (PPI)} analysis of correlative genes of these IncRNAs

A total of 4086 genes were dysregulated in PTC tissues, of which 409 were identified as lncRNAcorrelative genes. All the statistically significant results of functional and pathway enriched analysis were shown in Table 7. As for Gene Ontology (GO) terms, the top 5 significantly enriched terms were listed in Table 7 . The results of Kyoto Encyclopedia of Genes and Genomes (KEGG) analysis indicated that the related genes of

B

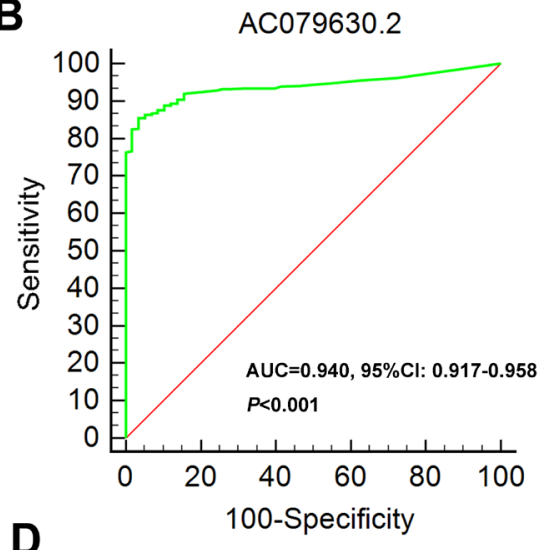

D

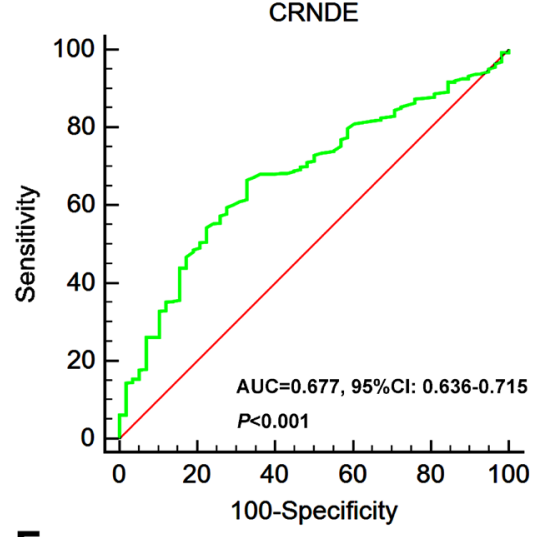

$\mathbf{F}$

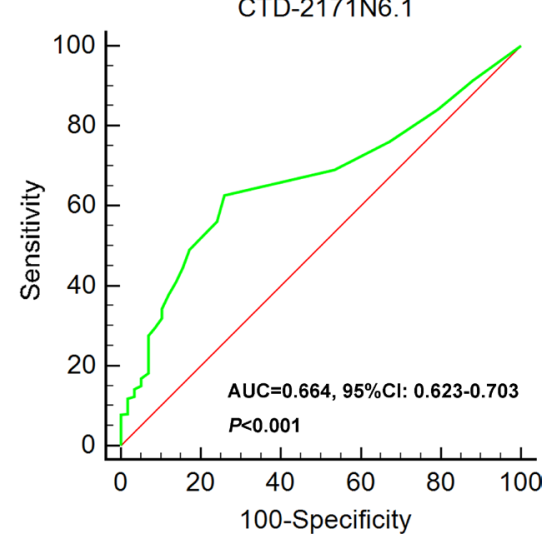

Figure 3: Diagnostic value of 3 novel IncRNAs to distinguish PTC and normal tissues. (A) Scatter diagram of AC079630.2; (B) ROC curve of AC079630.2; (C) Scatter diagram of CRNDE; (D) ROC curve of CRNDE; (E) Scatter diagram of CTD-2171N6.1; (F) ROC curve of CTD-2171N6.1. 


\section{Table 5: The expression data of 3 novel IncRNAs in GEO datasets}

\begin{tabular}{|c|c|c|c|c|c|c|c|c|c|c|c|}
\hline \multirow{2}{*}{ GEO datasets } & \multirow{2}{*}{ Year } & \multirow{2}{*}{ Country } & \multirow{2}{*}{ Platform } & \multirow{2}{*}{ Samples } & \multirow{2}{*}{$N$} & \multicolumn{6}{|c|}{ Relative expression of IncRNAs (mean \pm SEM) } \\
\hline & & & & & & AC079630.2 & $P$ & CRNDE & $P$ & CTD-2171N6.1 & $P$ \\
\hline \multirow[t]{2}{*}{ GSE33630 } & 2012 & Belgium & GPL570 & PTC & 15 & & & $8.679 \pm 0.3023$ & 0.187 & & \\
\hline & & & & Non-cancer & 3 & & & $7.714 \pm 0.2610$ & & & \\
\hline \multirow[t]{2}{*}{ GSE3467 } & 2005 & USA & GPL570 & PTC & 9 & & & $6.187 \pm 0.2225$ & 0.969 & & \\
\hline & & & & Non-cancer & 9 & & & $6.171 \pm 0.3353$ & & & \\
\hline \multirow[t]{2}{*}{ GSE35570 } & 2015 & Poland & GPL570 & PTC & 65 & & & $7.464 \pm 0.1764$ & $<0.001$ & & \\
\hline & & & & Non-cancer & 51 & & & $6.248 \pm 0.1801$ & & & \\
\hline \multirow[t]{2}{*}{ GSE3678 } & 2006 & USA & GPL570 & PTC & 7 & & & $8.550 \pm 0.2097$ & 0.002 & & \\
\hline & & & & Non-cancer & 7 & & & $7.563 \pm 0.1329$ & & & \\
\hline \multirow[t]{2}{*}{ GSE50901 } & 2014 & Brazil & GPL13607 & PTC & 48 & & & $0.141 \pm 0.1281$ & 0.214 & & \\
\hline & & & & Non-cancer & 4 & & & $-0.454 \pm 0.5862$ & & & \\
\hline \multirow[t]{2}{*}{ GSE53157 } & 2013 & Portugal & GPL570 & PTC & 15 & & & $8.679 \pm 0.3023$ & 0.187 & & \\
\hline & & & & Non-cancer & 3 & & & $7.714 \pm 0.2610$ & & & \\
\hline \multirow[t]{2}{*}{ GSE6004 } & 2006 & USA & GPL570 & PTC & 14 & & & $4.557 \pm 0.2859$ & 0.553 & & \\
\hline & & & & Non-cancer & 4 & & & $4.224 \pm 0.1183$ & & & \\
\hline \multirow[t]{2}{*}{ GSE60542 } & 2015 & Belgium & GPL570 & PTC & 33 & & & $7.089 \pm 0.1708$ & 0.002 & & \\
\hline & & & & Non-cancer & 30 & & & $6.322 \pm 0.1642$ & & & \\
\hline \multirow[t]{2}{*}{ GSE64912 } & 2015 & Italy & GPL11154 & PTC & 18 & $13.580 \pm 2.9320$ & 0.045 & $4.079 \pm 0.7280$ & 0.140 & $0.304 \pm 0.0926$ & 0.380 \\
\hline & & & & Non-cancer & 4 & $0.040 \pm 0.0209$ & & $1.651 \pm 0.3393$ & & $0.124 \pm 0.0400$ & \\
\hline \multirow[t]{2}{*}{ GSE83520 } & 2016 & USA & GPL16791 & PTC & 12 & $21.090 \pm 3.9450$ & $<0.001$ & $3.895 \pm 0.5877$ & 0.040 & $0.061 \pm 0.0232$ & 0.419 \\
\hline & & & & Non-cancer & 12 & $0.290 \pm 0.1210$ & & $2.395 \pm 0.3550$ & & $0.039 \pm 0.0127$ & \\
\hline
\end{tabular}

AC079630.2 were mostly enriched in the pathway of "Transcriptional misregulation in cancer" and the related genes of CTD-2171N6.1 were mostly enriched in the pathways of "ECM-receptor interaction", "Protein digestion and absorption", "Amoebiasis", "Focal adhesion" and "PI3K-Akt signaling pathway" (Figure 10A-10C). However, there was no pathway significantly enriched by the related genes of CRNDE as assessed by the pathway analyses above. The PPI network was showed in Figure 11. Moreover, with the rank of degree, the top 20 genes (COL1A1, DCN, COL1A2, MMP14, SDC1, FN1, COL3A1, COL5A1, TNC, SPARC, VCAN, AR, CD44, POSTN, THBS2, COL5A3, ERBB4, MMP13, NID1 and COL5A2) were identified as core genes which participate in the progression of PTC.

\section{DISCUSSION}

In this study, the genome-wide high-throughput RNA-sequencing data of lncRNA expression level of PTC were retrieved from TCGA dataset. Survival analysis revealed that three IncRNAs (AC079630.2, CRNDE and CTD-2171N6.1) were pronouncedly related to the patient survival. More importantly, a risk score calculated by the expression level of these three lncRNAs was an independent prognostic factor of PTC.
Recent researches have revealed that lncRNAs play critical roles in cancers, including thyroid cancer [25-27]. The clinical role of a certain lncRNAs has been well documented. For example, remarkably higher expression of HOX transcript antisense RNA (HOTAIR) was detected in PTC tissues versus normal tissues [28].The expression of antisense non-coding RNA in the INK4 locus (ANRIL) was also distinctly up-regulated in thyroid cancer tissues and cells, and closely related to histological grade and status of lymph node metastasis [29]. Similarly, the BRAF-activated non-coding RNA (BANCR) levels were prominently lower in PTC tumor tissues than in control tissues. Decreased BANCR levels were related to tumor size, the presence of multifocal lesions and advanced stage [30]. Furthermore, another lncRNA, PTCSC3, was strongly downregulated in PTC tissues [31]. Not many IncRNAs have been verified to be effective indicators for the progression or prognosis prediction. A functional IncRNA, SLC6A9-5:2, was found to be down-regulated in PTC and it was involved in the radioactive therapy resistance of PTC [32]. However, only clinical role of one single lncRNA was studied in aforementioned studies.

Profiling of lncRNAs based on the techniques of microarray or RNA-sequencing can greatly facilitate the discovery of novel lncRNA candidates in PTC. A genome-wide analysis of lncRNA expression profile with microarray in PTC with 62 cases revealed that 
3499 lncRNAs were distinctly expressed. Quantitative real time polymerase chain reaction (qRT-PCR) was employed to verify randomly 10 distinctly expressed lncRNAs, including TCONS_12_00010365, n386477, n340790, lnc-LLPH-2:1 and 261 NR_003225.2 being upregulated, and lnc-PSD4-1:14, n335550, lnc-KCMF1-2:1, lnc-PLA2R1-1:1 and ENST00000422494.1 being downregulated [19]. Another microarray study based on three patients of PTC indicated that 675 lncRNAs were distinctly expressed in PTC samples versus adjacent noncancerous samples. To confirm the microarray results, eight abnormally expressed lncRNAs were also randomly selected for detection with RT-qPCR with 36 cases, including 4 upregulated (ENST00000503723, ENST00000423539, uc003tab.3 and NR_073085) and 4 downregulated (ENST00000515275, ENST00000570022, uc003qef.1 and ENST00000427243) [20]. In addition to microarray, next-generation sequencing (NGS) in thyroid cancer allows concurrent high-throughput sequencing exploration of variable genetic changes and thus can offer a wide-ranging cognition of thyroid cancer biology [33]. RNA sequencing was performed to identify the changes to the transcriptome profile in 12 thyroid tissues compared to paired normal adjacent tissues. A total of 188 lncRNAs were detected to be abnormally expressed in $50 \%$ or more

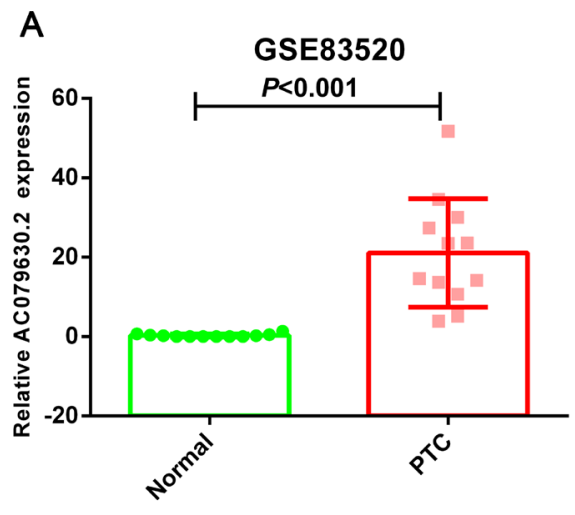

Tissue

C

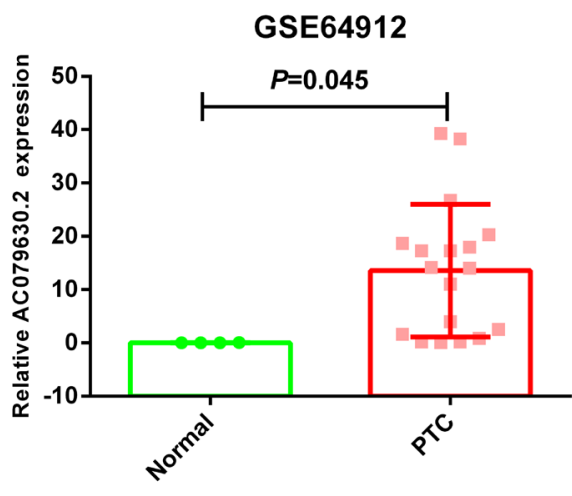

Tissue of the thyroid tissues as assessed by RNA-sequencing compared with paired normal adjacent tissues. Forty-seven lncRNAs were verified in 31 pairs of PTC specimens using RT-qPCR. The lncRNAs NONHSAT076747 and NONHSAT122730 were found to be related to lymph node metastasis, and NONHSAG051968 expression was adversely related to tumor size [21]. Whole exome sequencing was also performed by another group with 91 pairs of PTC tissues. They further performed Sanger sequencing with 311 pairs of samples. Interestingly, they found that lncRNA GAS8-AS1 is the second most commonly differentiated gene and functions as a new tumor suppressive IncRNA in thyroid [22]. However, most of the studies above were performed based on small number of patients. Due to different technique platforms, distinct setting for cut-off value, various patient sources, the profiling of differentially expressed lncRNAs was inconsistent. Furthermore, the prognostic role of these lncRNAs has not been explored.

In the light of the established molecular aberrations, the TCGA dataset has accomplished a comprehensive genetic and epigenetic investigation into a large cohort of thyroid cases by the most innovative approaches of next-generation sequencing [34-36]. To the best of our knowledge, there has been only one paper so far, which
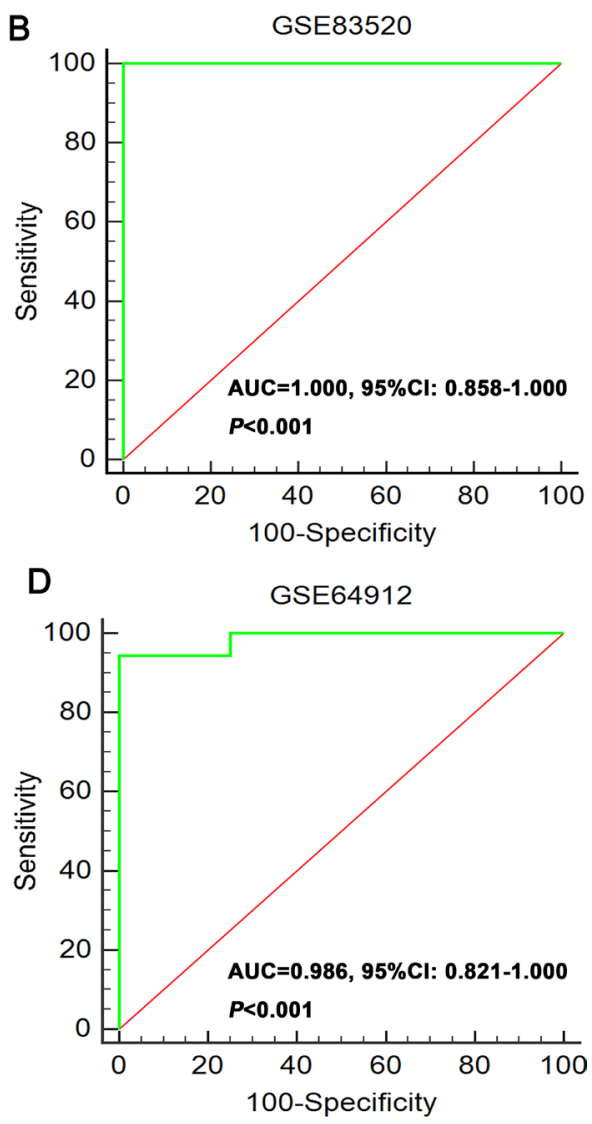

Figure 4: The diagnostic value of AC079630.2 to distinguish PTC and normal tissues. (A) Scatter diagram of GSE64912; (B) ROC curve of GSE64912; (C) Scatter diagram of GSE83520; (D) ROC curve of GSE83520. 
has mined the high-throughput data of lncRNAs of PTC and was published when we were preparing for our paper. Ma et al. [24] reported that 220 lncRNAs were altered expressed from the annotated 2773 lncRNAs which were approved by the HUGO gene nomenclature committee in TCGA dataset. Among these lncRNAs, FAM41C,
A

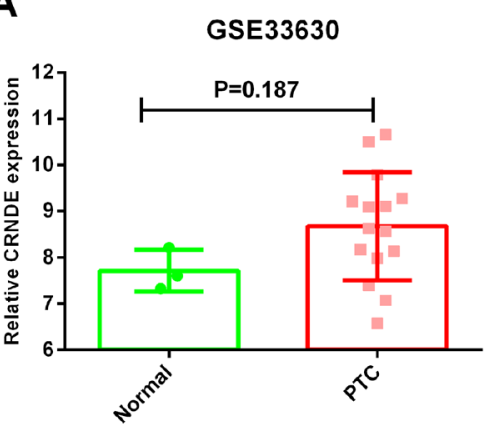

Tissue

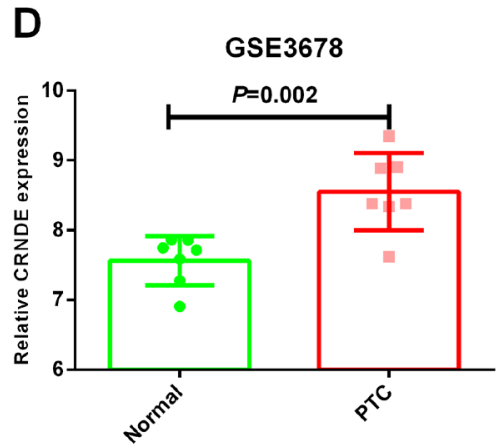

Tissue
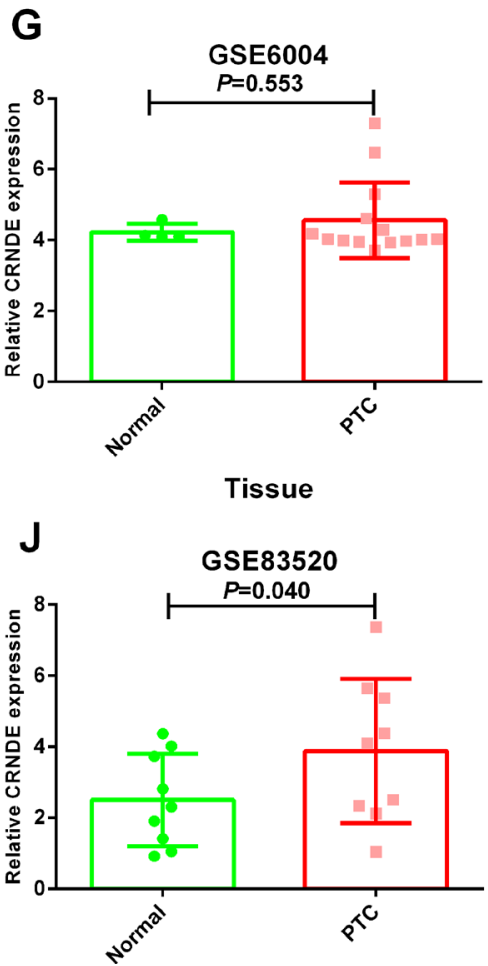

B

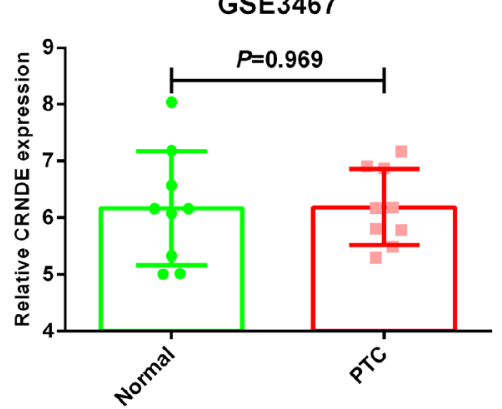

Tissue

E

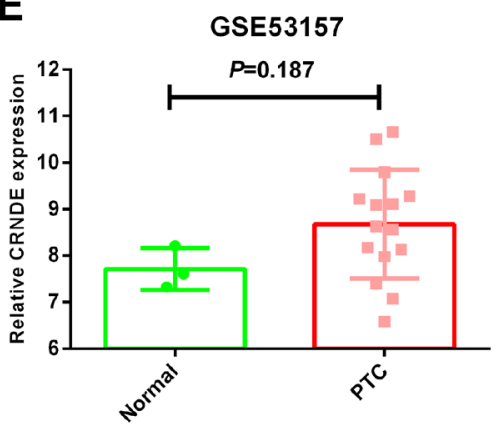

Tissue

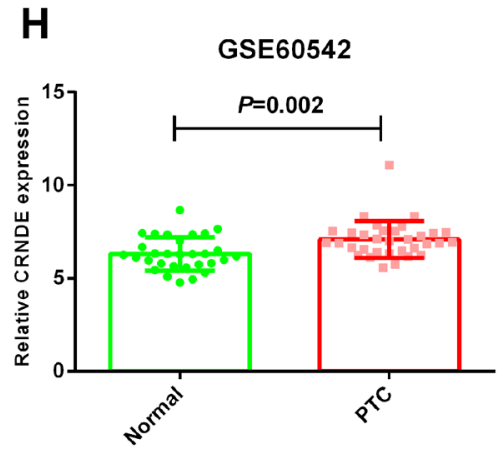

Tissue

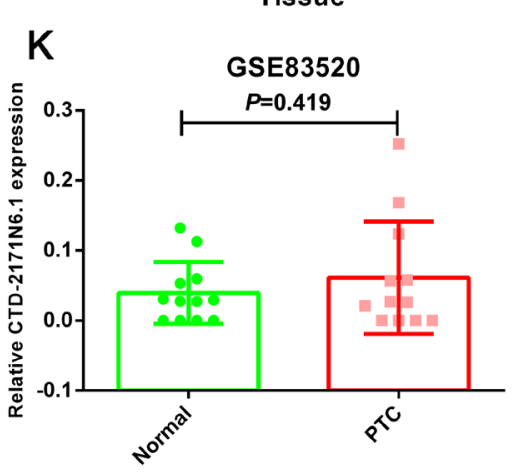

C

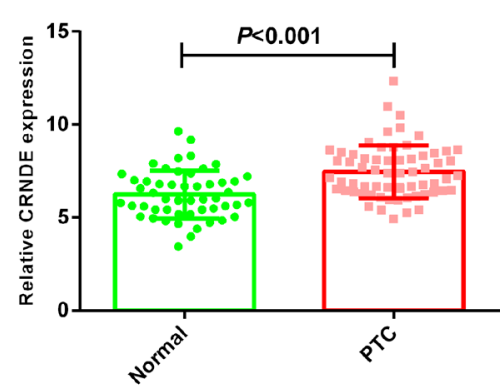

Tissue

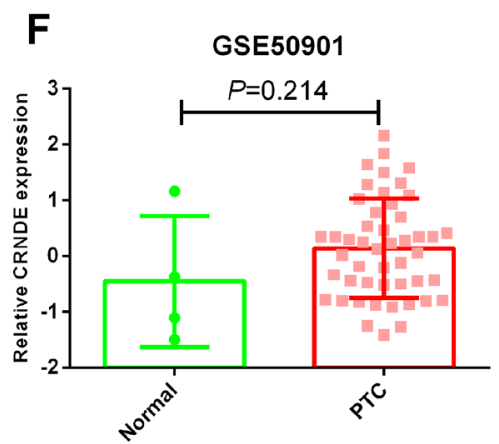

Tissue
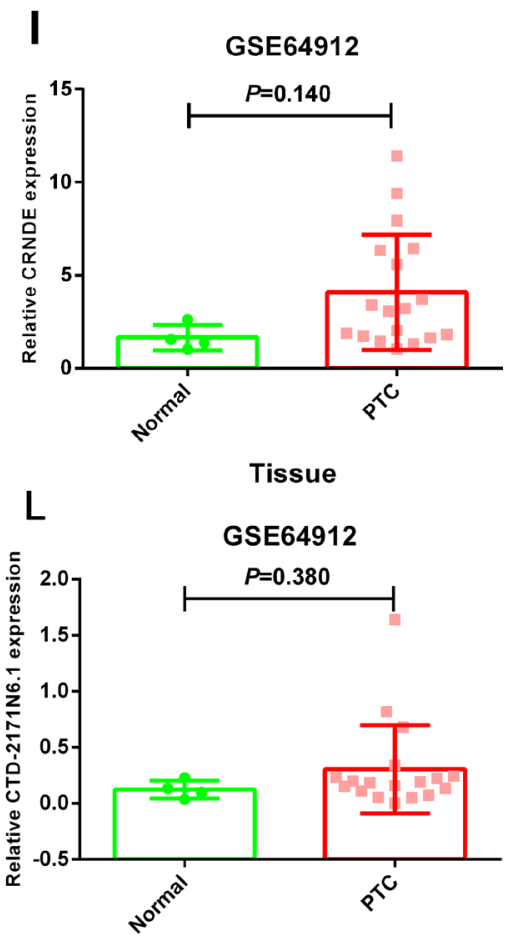

Figure 5: Differential expression of CRNDE and CTD-2171N6.1 in PTC tissues compared to normal tissues. (A) CRNDE: GSE33630; (B) CRNDE: GSE3467; (C) CRNDE: GSE35570; (D) CRNDE: GSE3678; (E) CRNDE: GSE53157; (F) CRNDE: GSE50901; (G) CRNDE: GSE6004; (H) CRNDE: GSE60542; (I) CRNDE: GSE64912; (J) CRNDE: GSE83520; (K) CTD2171N6.1: GSE83520 (L) CTD-2171N6.1: GSE64912. 
A
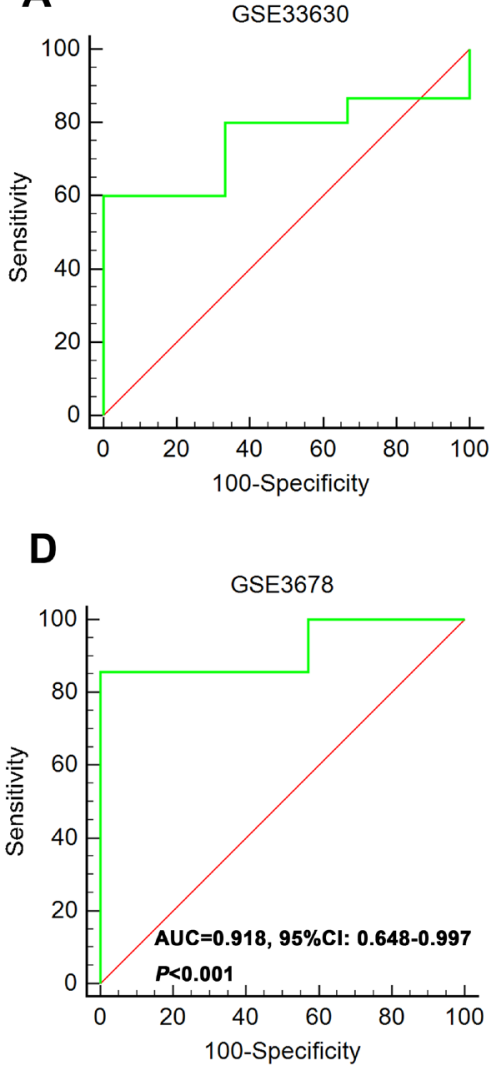

G

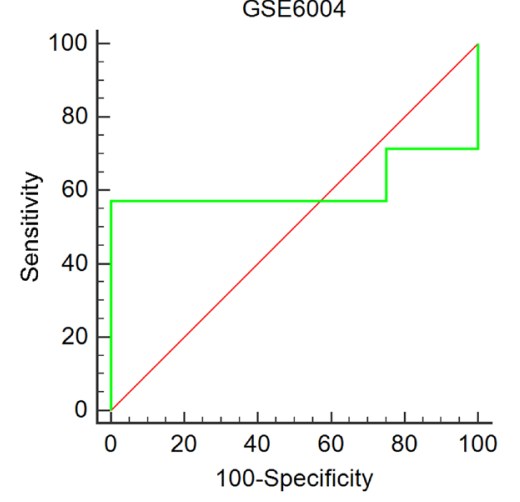

$\mathbf{J}$

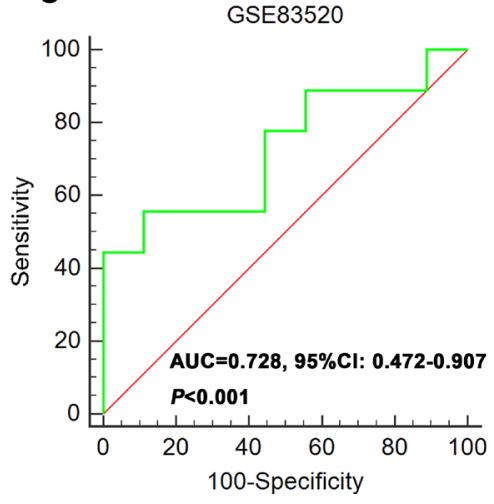

B

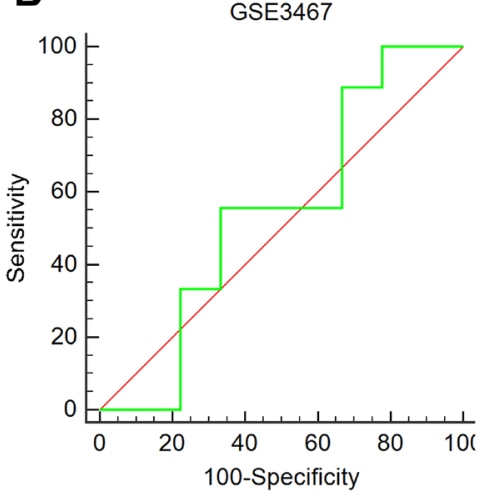

E

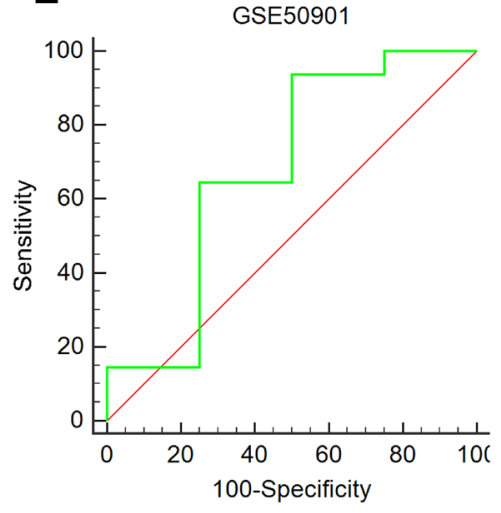

H

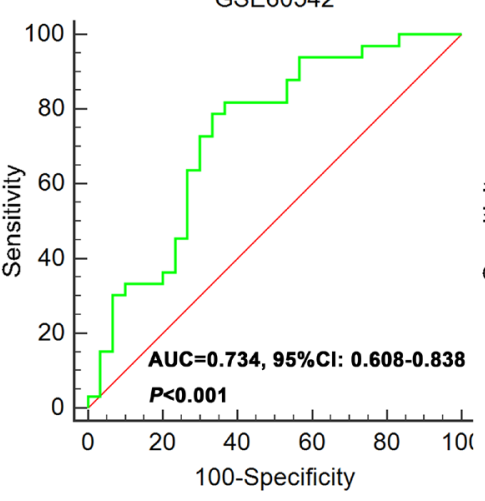

C GSE35570
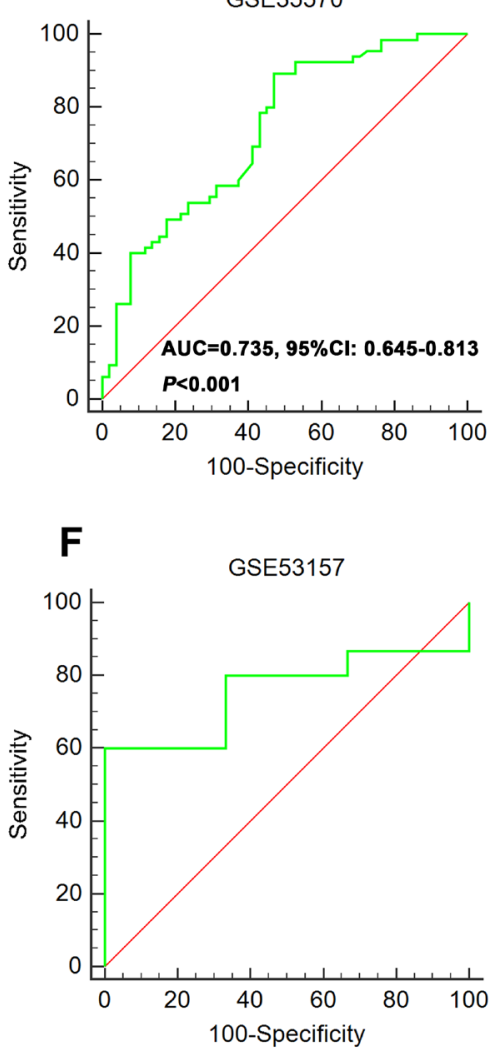

I

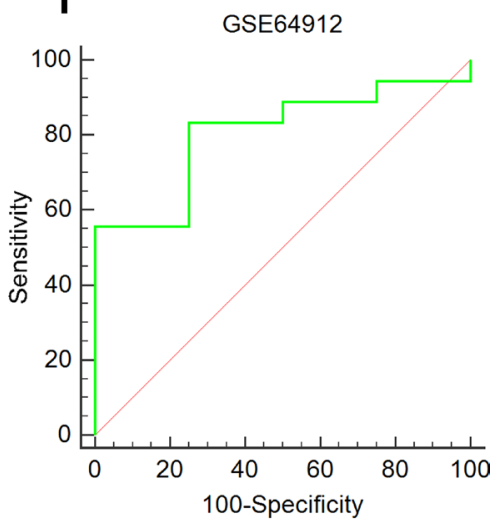

Figure 6: ROC curve of CRNDE to distinguish PTC and normal tissues. (A) GSE33630; (B) GSE3467; (C) GSE35570; (D) GSE3678; (E) GSE50901; (F) GSE53157; (G) GSE6004; (H) GSE60542; (I) GSE64912; (J) GSE83520. 

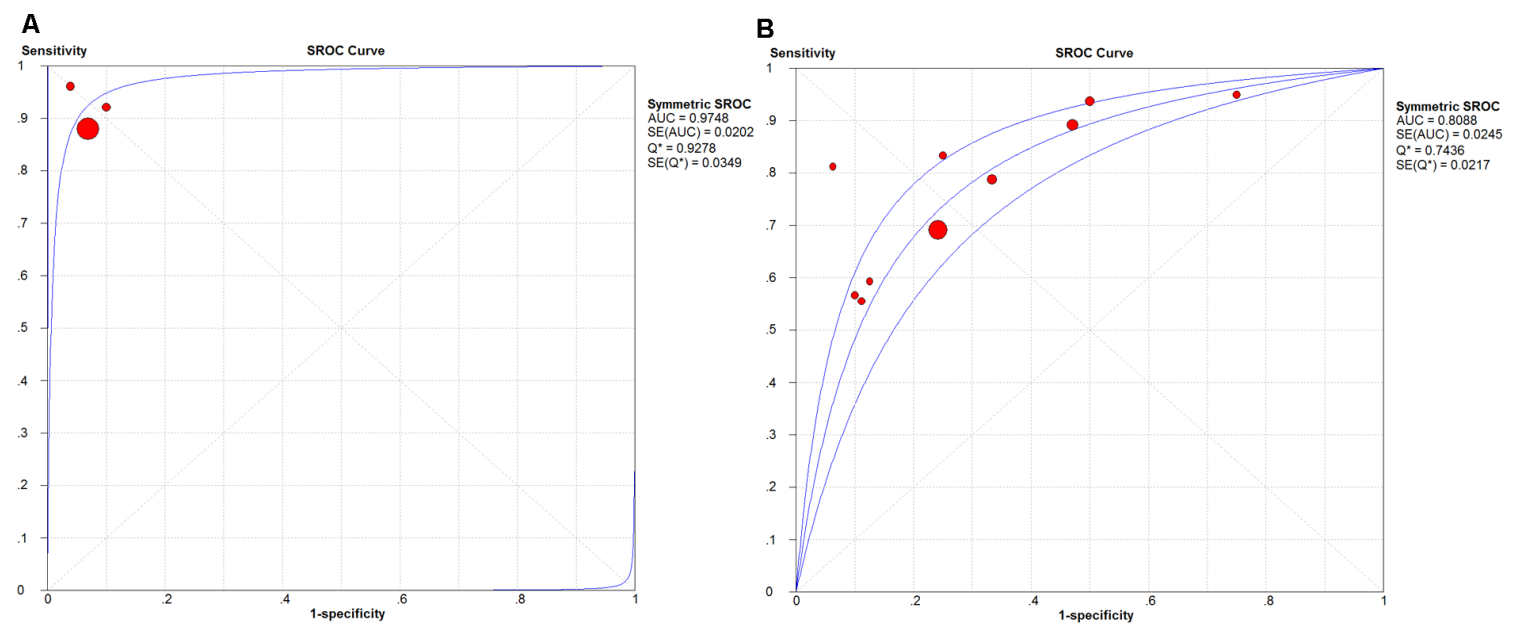

Figure 7: The SROC curve of AC079630 .2 and CRNDE. (A) AC079630.2; (B) CRNDE.

A

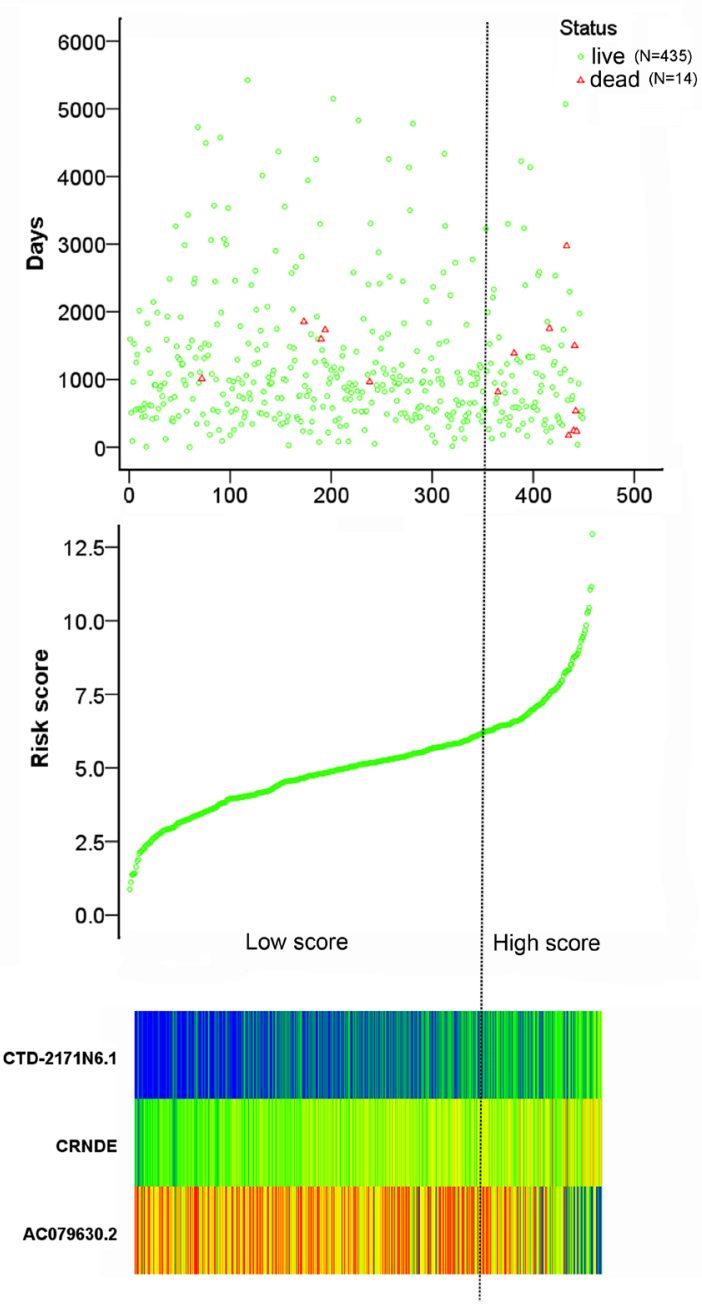

B Risk score
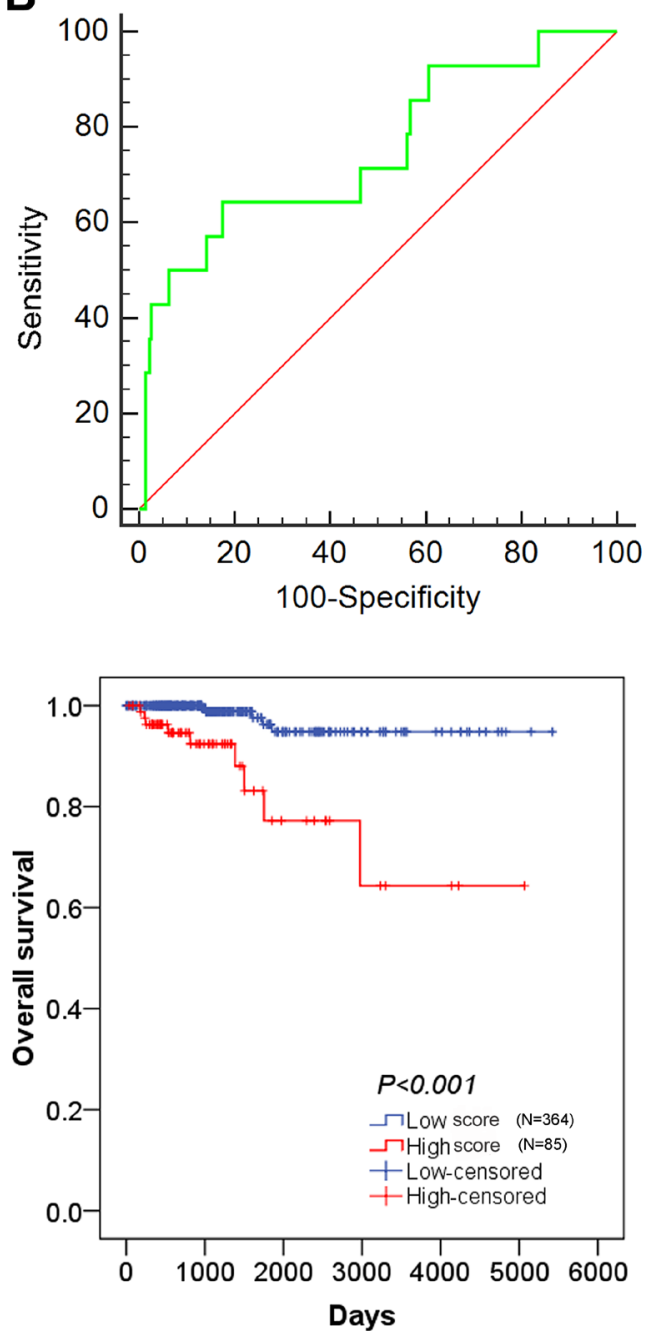

Figure 8: The risk score analysis of 449 PTC patients. (A) top: survival status of PTC patients; middle: risk score of lncRNAs signature; bottom: risk score of 3 lncRNAs; (B) top: the diagnostic significance of risk score to survival outcome; bottom: the association of risk score with OS. 
Table 6: The univariate and multivariate analysis of clinicopathological parameters and prognostic model for OS in PTC patients (TCGA dataset, $N=449$ )

\begin{tabular}{ccccc}
\hline \multirow{2}{*}{ Groups } & \multicolumn{2}{c}{ Univariate analysis } & \multicolumn{2}{c}{ Multivariate analysis } \\
\cline { 2 - 5 } & HR (95\% CI) & $\boldsymbol{P}$ & HR (95\% CI) & $\boldsymbol{P}$ \\
\hline Age $^{\mathrm{a}}$ & $1.121(1.076-1.167)$ & $<\mathbf{0 . 0 0 1}$ & $1.149(1.074-1.229)$ & $<\mathbf{0 . 0 0 1}$ \\
Gender & $0.791(0.247-2.531)$ & 0.692 & $1.647(0.239-11.352)$ & 0.613 \\
Tumor subtypes & $0.489(0.165-1.452)$ & 0.198 & $0.306(0.070-1.339)$ & 0.116 \\
History of other tumor & $4.163(1.158-14.971)$ & $\mathbf{0 . 0 2 9}$ & $2.751(0.370-20.445)$ & 0.323 \\
Pathological stage & $2.683(1.585-4.540)$ & $<\mathbf{0 . 0 0 1}$ & $1.523(0.491-4.723)$ & 0.466 \\
T stage & $3.153(0.985-10.089)$ & 0.053 & $0.745(0.031-18.065)$ & 0.856 \\
N stage & $1.421(0.492-4.103)$ & 0.516 & $0.456(0.087-2.399)$ & 0.354 \\
M stage & $5.258(1.164-23.755)$ & $\mathbf{0 . 0 3 1}$ & $0.748(0.048-11.568)$ & 0.835 \\
Focus types & $0.283(0.063-1.273)$ & 0.100 & $0.115(0.009-1.414)$ & 0.091 \\
Extrathyroidal extension & $2.660(0.885-7.992)$ & 0.081 & $0.757(0.049-11.676)$ & 0.842 \\
BRAFv600 & $0.830(0.274-2.517)$ & 0.743 & $1.664(0.321-8.633)$ & 0.544 \\
Risk score & $8.592(2.878-25.651)$ & $<\mathbf{0 . 0 0 1}$ & $7.092(1.667-30.179)$ & $\mathbf{0 . 0 0 8}$ \\
\hline
\end{tabular}

HR: hazard ratio; CI: confidence interval; ${ }^{a}$ continuous.

CTBP1-AS2, LINC00271, HAR1A, LINC00310 and HAS2-AS1 were found to be related to patient recurrence. After the classical clinicopathological factors and $\mathrm{BRAF}^{\mathrm{V} 600 \mathrm{E}}$ mutation were adjusted, LINC00271 was proved to be an independent risk factor for a series of clinical characteristics, including extrathyroidal extension, lymph node metastasis, advanced tumor stage III/IV and recurrence in multivariate analyses. The results of our study were different with that published by Ma et al. The reasons might be that Ma et al. [24] carried out the expression alteration of the lncRNAs using The cBioPortal for Cancer Genomics, while in the current study, DEseq $\mathrm{R}$ package was used, which is widely applied for the analysis of RNA-seq data. Furthermore, we annotated 7589 lncRNAs, more than Ma et al. did, using Ensembl genome browser (www.ensembl.org/), which supports the result of our current study. Collectively, based on 507 thyroid patients and 59 normal thyroid tissues, we identified 734 differentially expressed lncRNAs, among which 33 lncRNAs were evaluated to be significantly associated with OS of thyroid patients by univariate analysis and eventually, the prognostic values of three lncRNAs (AC079630.2, CRNDE and CTD-2171N6.1) were confirmed by multivariate analysis. Generally, DFS is more appropriate to assess the outcome of PTC patients. Actually, we also investigated the association of these IncRNAs with DFS, but the results indicated that only CRNDE was significantly associated with DFS. A specific risk score was calculated with these three lncRNAs and this risk score and age were identified as independent prognostic indicators for PTC. In addition, the risk score was positively correlated with age, which indicated that the elder PTC patients had higher risk score. Furthermore, the aberrant expression of AC079630.2 and CRNDE could be confirmed with other PTC samples based on different detecting methods, namely, microarrays, which strengthens our current findings. We also attempted to validate the prognostic value of the three-lncRNA signature in PTC based on GEO datasets. Regrettably, no survival data of AC079630.2, CRNDE or CTD-2171N6.1 in PTC was available in GEO datasets. The genetic alteration of lncRNAs was observed in various tumors and its alteration can assist to predict patients' survival $[37,38]$. Nevertheless, no alteration was observed for CRNDE and no relevant information about another two IncRNAs could be achieved in cBioPortal for Cancer Genomics (http://www.cbioportal.org/). Other clinical experiments need to be carried out to verify the prognostic role of the novel three-lncRNA signature in PTC.

Concerning the biological function and clinical role of these IncRNAs, only CRNDE has been studied in cancers so far. Several studies indicated that CRNDE expression significantly increased in gliomas and was related to the tumor progression, recurrence and survival $[39,40]$. CRNDE can promote the malignant progress of glioma by lessening miR-384/PIWIL4/STAT3 Axis, miR-186 or mTOR signaling pathways [41-43]. CRNDE has been reported to be a new serum-based marker for diagnosis and prognosis of colorectal cancer [44], and it can stimulate cell growth and chemoresistance of colorectal cancer cells via correlating with IRX5 or miR$181 \mathrm{a}-5 \mathrm{p}$-mediated regulation of $\mathrm{Wnt} / \beta$-catenin signaling $[45,46]$. Moreover, CRNDE can promote tumor growth in medulloblastoma [47], hepatic carcinoma [48], gallbladder carcinoma [49], renal cell carcinoma [50] as well as in ovarian carcinoma patients [51] with various prospective molecular mechanisms. However, the clinical value and underlying mechanism of CRNDE in PTC remains largely 
unveiled and no possible pathway could be suggested by our signaling investigation. More exploration is acquired to understand the mechanism of CRNDE in the future. The pathway of "Transcriptional misregulation in cancer" is closely related to AC079630.2 and 5 pathways are linked to CTD-2171N6.1. Interestingly, published studies indicate that some genes can exert its oncogenic function by activating PI3K-Akt signaling pathway in PTC $[52,53]$, which can be functioned via CTD-2171N6.1. However, relevant validated research needs to be carried out in the future.
One of the limitations of the current study is that most of the cases in the cohort were censored and there were only 14 end events, which might cause some bias to obtain accurate result. Furthermore, no data on the iodine therapy or other details of therapeutic strategies could be achieved. Finally, validation with FISH or qPCR is still needed afterwards.

To sum up, we have identified three novel lncRNAs (AC079630.2, CRNDE and CTD-2171N6.1) which are markedly related to the survival of PTC. The novelty of the current study lies in the special risk score generated by
A

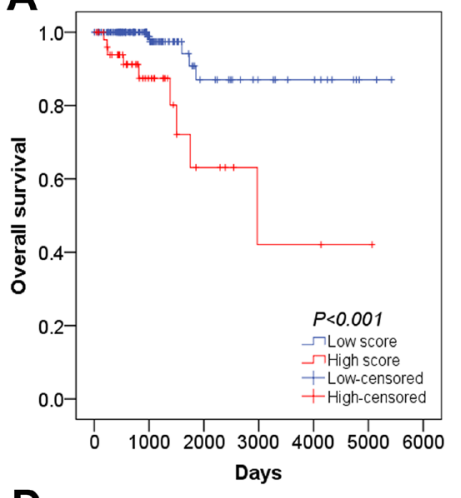

D

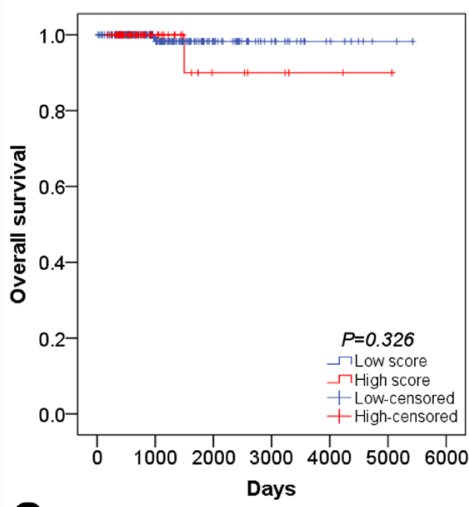

G

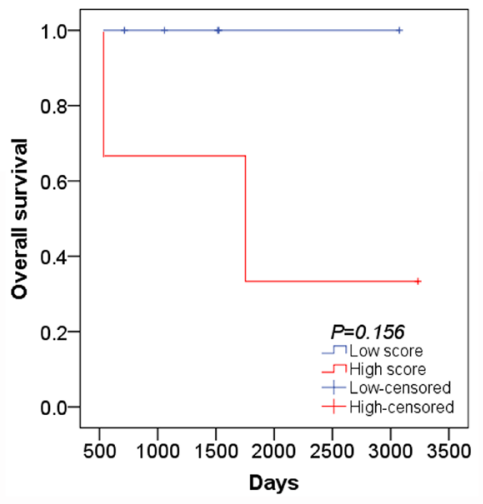

B

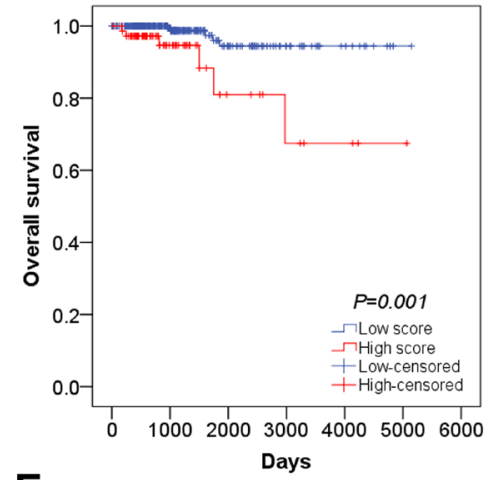

E

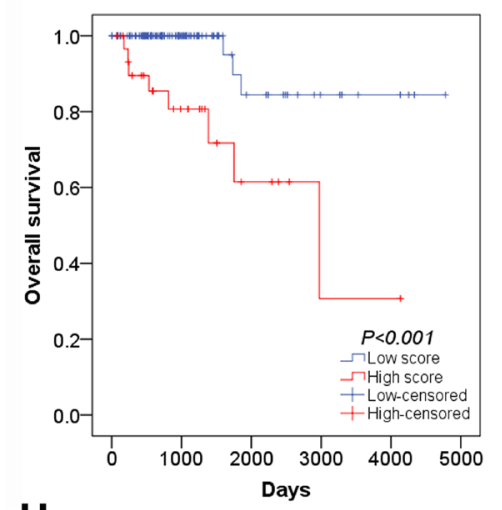

H

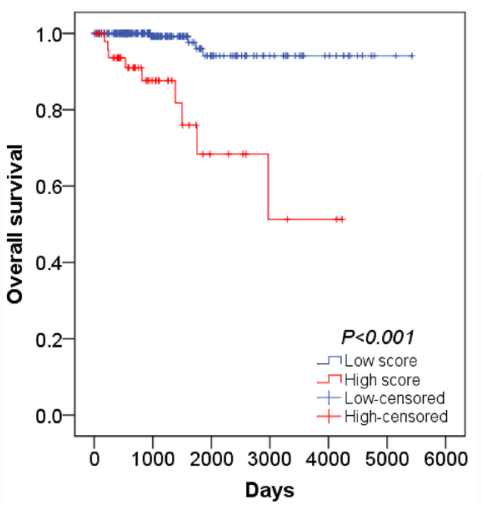

C

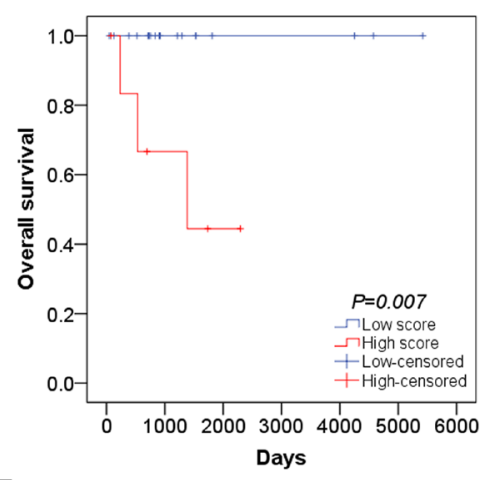

F

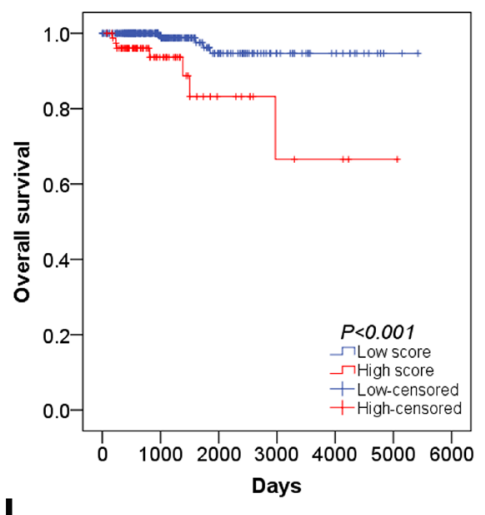

I

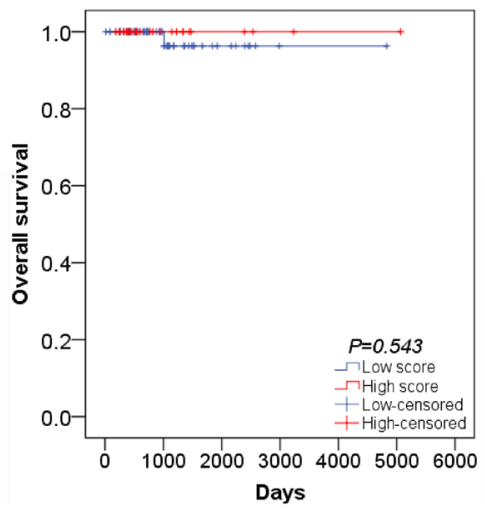

Figure 9: Subgroup analysis of the association between risk score and survival outcome. (A) Kaplan-Meier survival curve in elder patients; (B) Kaplan-Meier survival curve in patients without the other tumor; (C) Kaplan-Meier survival curve in patients with the other tumor; (D) Kaplan-Meier survival curve in early stage patients; (E) Kaplan-Meier survival curve in advanced stage patients; (F) Kaplan-Meier survival curve in M0 stage patients; (G) Kaplan-Meier survival curve in M1 stage patients; (H) Kaplan-Meier survival curve in classical/usual PTC patients; (I) Kaplan-Meier survival curve in follicular PTC patients. 
$\mathrm{AC079630.2}$

GO terms (BP)

GO:0045165 cell fate commitment

GO:0030318 melanocyte differentiation

GO:0035556 intracellular signal transduction

GO:0046777 protein autophosphorylation

GO:0007275 multicellular organism development

\section{GO terms (CC)}

GO:0016021 integral component of membrane

GO:0005886 plasma membrane

GO:0098794 postsynapse

GO:0030054 cell junction

GO:0005794 Golgi apparatus

\section{GO terms (MF)}

GO:0004714 transmembrane receptor protein tyrosine kinase activity

GO:0005154 epidermal growth factor receptor binding

GO:0005524 ATP binding

GO:0005088 Ras guanyl-nucleotide exchange factor activity

GO:0030276 clathrin binding

\section{KEGG analysis}

hsa05202:Transcriptional misregulation in cancer

\section{CRNDE}

\section{GO terms (BP)}

GO:0001755 neural crest cell migration

GO:0007399 nervous system development

GO:0042493 response to drug

GO:0030206 chondroitin sulfate biosynthetic process

GO:0048843 negative regulation of axon extension involved in axon guidance

\section{GO terms (CC)}

GO:0005576 extracellular region

GO:0005615 extracellular space

GO:0043198 dendritic shaft

$\begin{array}{lll}5 & 2.00 \mathrm{E}-04 & 3.09 \mathrm{E}-01 \\ 4 & 2.58 \mathrm{E}-04 & 3.99 \mathrm{E}-01 \\ 9 & 4.00 \mathrm{E}-03 & 6.03 \mathrm{E}+00 \\ 6 & 4.69 \mathrm{E}-03 & 7.03 \mathrm{E}+00 \\ 10 & 5.70 \mathrm{E}-03 & 8.48 \mathrm{E}+00\end{array}$

$\begin{array}{cll}11 & 1.64 \mathrm{E}-02 & 1.61 \mathrm{E}+01 \\ 9 & 3.96 \mathrm{E}-02 & 3.49 \mathrm{E}+01 \\ 2 & 8.91 \mathrm{E}-02 & 6.29 \mathrm{E}+01\end{array}$




\section{GO terms (MF)}

GO:0030215 semaphorin receptor binding

GO:0045499 chemorepellent activity

$\begin{array}{lll}2 & 5.83 \mathrm{E}-02 & 5.05 \mathrm{E}+01 \\ 2 & 6.81 \mathrm{E}-02 & 5.62 \mathrm{E}+01 \\ 2 & 9.21 \mathrm{E}-02 & 6.78 \mathrm{E}+01\end{array}$

GO:0008146 sulfotransferase activity

\section{CTD-2171N6.1}

\section{GO terms (BP)}

GO:0030198 extracellular matrix organization

$6.26 \mathrm{E}-21$

GO:0030199 collagen fibril organization

GO:0030574 collagen catabolic process

GO:0001501 skeletal system development

1.52E-09

2.29E-06

GO:0007155 cell adhesion

\section{GO terms (CC)}

GO:0005578 proteinaceous extracellular matrix

$\begin{array}{lll}25 & 7.15 \mathrm{E}-21 & 8.27 \mathrm{E}-18 \\ 24 & 1.23 \mathrm{E}-18 & 1.42 \mathrm{E}-15 \\ 43 & 7.28 \mathrm{E}-16 & 8.99 \mathrm{E}-13 \\ 35 & 2.50 \mathrm{E}-12 & 2.89 \mathrm{E}-09 \\ 12 & 1.81 \mathrm{E}-11 & 2.09 \mathrm{E}-08\end{array}$

GO:0031012 extracellular matrix

$1.81 \mathrm{E}-11$

2.09E-08

\section{GO terms (MF)}

GO:0005201 extracellular matrix structural constituent

$$
11
$$

$1.25 \mathrm{E}-11$

$1.55 \mathrm{E}-08$

GO:0004222 metalloendopeptidase activity

GO:0005518 collagen binding

9

$5.90 \mathrm{E}-07$

7.33E-04

GO:0005509 calcium ion binding

7

GO:0008201 heparin binding

\section{KEGG analysis}

hsa04512:ECM-receptor interaction

GO: Gene Ontology; KEGG: Kyoto Encyclopedia of Genes and Genomes; BP biological process; CC cellular component; MF molecular function.

the three lncRNAs, which is an independent prognostic indicator for PTC. The accurate molecular roles of these three lncRNAs identified warrants further characterization, but our current data propose that they are prospective to play pivotal roles in PTC tumorigenesis and more importantly, these novel lncRNAs are closely related to patients' survival. These results have far-reaching consequences with respect to molecular prediction PTC.

\section{MATERIALS AND METHODS}

\section{Patient cohort and selection of candidate IncRNAs}

The RNA-sequencing and clinical data of 510 PTC patients and 59 normal thyroid tissues were provided by
TCGA data center (https://portal.gdc.cancer.gov/). After choosing the maximum expression value when multiple detections were performed for the same patient, 507 PTC patients and 59 normal thyroid tissues were finally included. The type of surgery and whether these patients received radioactive iodine therapy were unclear and the follow-up data was available for 502 patients. To further analyze the association of genes level with clinical data, the expression of genes were $\log 2$ transformed and records were considered as censored when the expression level were 0 or 1 [54]. In addition, the genes were eliminated when the censored data were more than $10 \%$. Dysregulated genes were screened by R software using the package "DEseq" and genes with significant differential expression between PTC tissues and normal thyroid tissues were selected by the standard of $\log 2$ fold change 

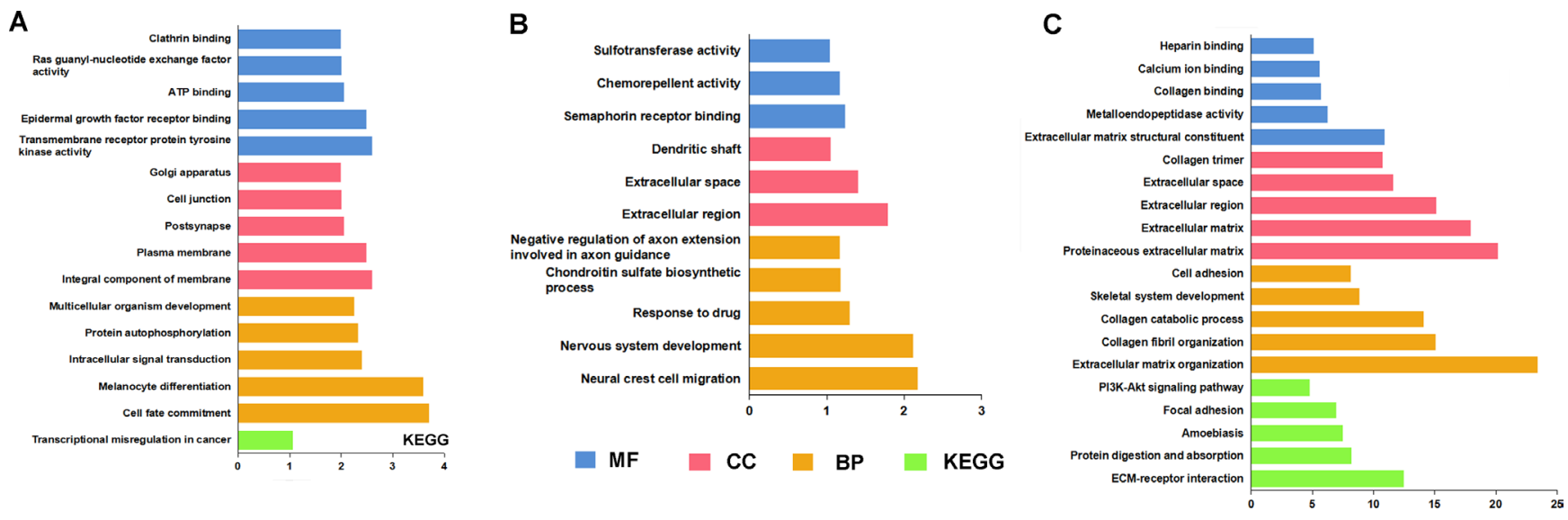

Figure 10: GO terms and KEGG pathways analysis by target genes of 3 IncRNAs. (A) AC079630.2; (B) CRNDE; (C) CTD2171N6.1.

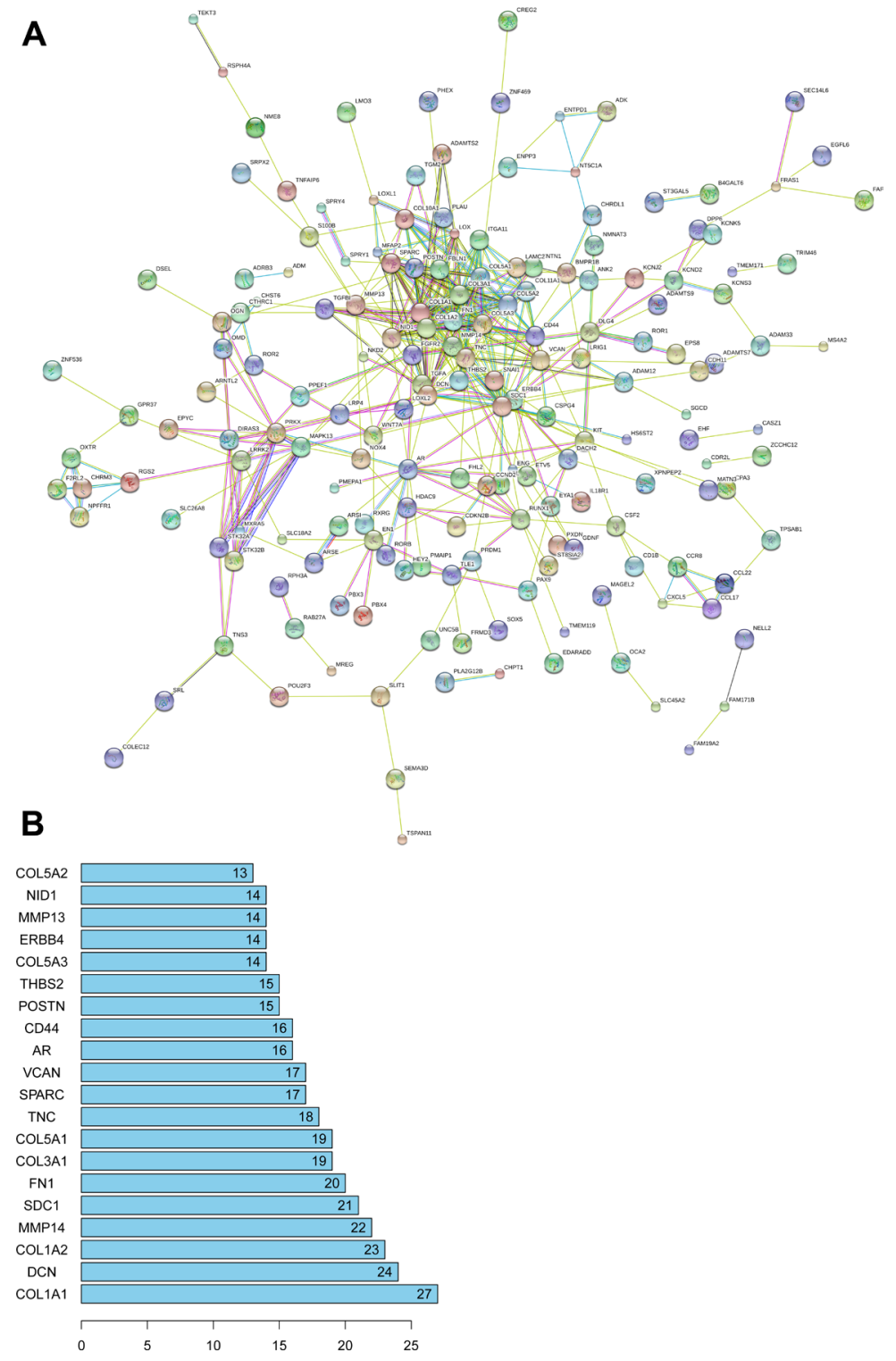

Figure 11: PPI network of 408 target genes of 3 novel IncRNAs. (A) map of PPI network; (B) core genes of PPI network. 
(FC) and adjusted $P$ value. The genes were noted as upregulated or down-regulated when $\operatorname{logFC}>1$ or $\operatorname{logFC}<-1$ with adjusted $P<0.05$, respectively. Subsequently, the expression data of dysregulated lncRNAs and survival data were analyzed with univariate Cox proportional hazards regression model. To explore each lncRNA as an independent indicator for survival, multivariate stepwise regression analysis was utilized for lncRNAs which were significantly associated with $\mathrm{OS}(P<0.05)$ in univariate analysis. Finally, the diagnostic and prognostic values of those selected IncRNAs were validated using other cohorts from Gene Expression Omnibus (GEO) databases and a meta-analysis was subsequently conducted via the calculation of summarized ROC (sROC).

\section{Construction of IncRNA-related prognostic indicator}

With the coefficient value of multivariate analysis, an IncRNA-related prognostic model was constructed by calculating a risk score for each subject. The risk score $=\beta$ of $\operatorname{lncRNA} 1 *$ level of $\operatorname{lncRNA} 1+\beta$ of $\operatorname{lncRNA} 2 *$ level of lncRNA2 $+\ldots+\beta$ of lncRNAn * level of lncRNAn. Additionally, patients were divided into the groups of high risk score and low risk score with the optimal cut off value. And the risk score was regarded as a biomarker for further analysis. Moreover, multivariate Cox proportional hazards regression was performed to adjust classical clinicopathological parameters, such as pathological stage, $T$ stage, $N$ stage, $M$ stage, focus types, extrathyroidal extension and BRAF ${ }^{\mathrm{V} 600 \mathrm{E}}$.

\section{Functional and protein-protein interaction (PPI) analysis for the correlative genes of IncRNAs}

Pearson correlation analysis was performed to explore the correlation between dysregulated genes and each lncRNA in prognostic model. According to the ranked coefficients, the top $1 \%$ genes were selected as lncRNAassociated genes for further analysis. Then, GO and KEGG enriched analyses, which are functional annotation analyses by bioinformatics enrichment tools using large gene lists [55], were conducted using the related genes of each lncRNA separately in DAVID website (http:// david.abcc.ncifcrf.gov/). PPI was performed within the Interacting Genes/Proteins (STRING) database (http:// string-db.org/).

\section{Statistical analysis}

HR and corresponding 95\% CI were assessed by univariate/multivariate Cox proportional hazards regression model to explore the prognostic impact of clinicopathological parameters and biomarkers. KaplanMeier survival curve and log-rank test were conducted to investigate the association between risk score and OS in PTC patients. In addition, we performed a subgroup analysis with clinicopathological parameters which were correlated with OS and subtypes of PTC. ROC curve was implemented to estimate the optimal cut off value of risk score to predict survival status. Simultaneously, Spearman rank correlation was performed to assess the association between three lncRNAs and clinicopathological parameters. Statistical significance threshold was set at a two-side $P<$ 0.05 . The analyses above were conducted with $\mathrm{R}$ software and SPSS 22.0 (SPSS Inc., Chicago, IL, USA).

\section{ACKNOWLEDGMENTS AND FUNDING}

The authors thank TCGA for providing the data. The study is funded by Guangxi Scientific Research and Technology Development Plan (1598011-4), the National Natural Science Foundation of China (NSFC 81560448), Guangxi Medical University Training Program for Distinguished Young Scholars (2017) and Guangxi Health Bureau Research Project (Z2012053).

\section{CONFLICTS OF INTEREST}

The authors declare no competing financial interests.

\section{REFERENCES}

1. Broecker-Preuss M, Becher-Boveleth N, Muller S, Mann K. The BH3 mimetic drug ABT-737 induces apoptosis and acts synergistically with chemotherapeutic drugs in thyroid carcinoma cells. Cancer Cell Int. 2016; 16:27.

2. Verburg FA, Van Santen HM, Luster M. Pediatric papillary thyroid cancer: current management challenges. Onco Targets Ther. 2016; 10:165-75.

3. Thyroid Cancer Treatment (PDQ(R)): Health Professional Version. PDQ Cancer Information Summaries. (Bethesda (MD). 2002.

4. Shen X, Miao ZM, Lu W, Gu DL, Yang D, Shen H, Geng F. Clinical experience with modified Miccoli's endoscopic thyroidectomy for treatment of thyroid carcinoma in 86 cases. Eur J Med Res. 2013; 18:51.

5. Samsonov R, Burdakov V, Shtam T, Radzhabova Z, Vasilyev D, Tsyrlina E, Titov S, Ivanov M, Berstein L, Filatov M, Kolesnikov N, Gil-Henn H, Malek A. Plasma exosomal miR-21 and miR-181a differentiates follicular from papillary thyroid cancer. Tumour Biol. 2016; 37:12011-21.

6. Maeda H, Kutomi G, Satomi F, Shima H, Mori M, Hirata K, Takemasa I. Clinicopathological characteristics of thyroid cancer misdiagnosed by fine needle aspiration. Exp Ther Med. 2016; 12:2766-2772.

7. He Y, Pan MZ, Huang JM, Xie P, Zhang F, Wei LG. Iodine-131: An Effective Method for Treating Lymph Node Metastases of Differentiated Thyroid Cancer. Med Sci Monit. 2016; 22:4924-4928. 
8. Ming J, Liu Z, Zeng W, Maimaiti Y, Guo Y, Nie X, Chen C, Zhao X, Shi L, Liu C, Huang T. Association between BRAF and RAS mutations, and RET rearrangements and the clinical features of papillary thyroid cancer. Int J Clin Exp Pathol. 2015; 8:15155-15162.

9. Li J, Zhang S, Zheng S, Zhang D, Qiu X. The BRAF V600E mutation predicts poor survival outcome in patients with papillary thyroid carcinoma: a meta analysis. Int J Clin Exp Med. 2015; 8:22246-22253.

10. Bullock M, Lim G, Li C, Choi IH, Kochhar S, Liddle C, Zhang L, Clifton-Bligh RJ. Thyroid transcription factor FOXE1 interacts with ETS factor ELK1 to co-regulate TERT. Oncotarget. 2016; 7:85948-85962. https://doi. org/10.18632/oncotarget.13288.

11. Liu C, Liu Z, Chen T, Zeng W, Guo Y, Huang T. TERT promoter Mutation and Its Association with Clinicopathological Features and Prognosis of Papillary Thyroid Cancer: A Meta-analysis. Sci Rep. 2016; 6:36990.

12. Xu B, Tuttle RM, Sabra M, Ganly I, Ghossein RM. Primary Thyroid Carcinoma with Low-Risk Histology and Distant Metastases: Clinico-Pathologic and Molecular Characteristics. Thyroid. 2017; 27:632-40.

13. Su X, Jiang X, Wang W, Wang H, Xu X, Lin A, Teng X, Wu H, Teng L. Association of telomerase reverse transcriptase promoter mutations with clinicopathological features and prognosis of thyroid cancer: a meta-analysis. Onco Targets Ther. 2016; 9:6965-76.

14. Fu QF, Pan PT, Zhou L, Liu XL, Guo F, Wang L, Sun H. Clinical significance of preoperative detection of serum p53 antibodies and BRAF(V600E) mutation in patients with papillary thyroid carcinoma. Int J Clin Exp Med. 2015; 8:21327-21334.

15. Vuong HG, Altibi AM, Abdelhamid AH, Ngoc PU, Quan VD, Tantawi MY, Elfil M, Vu TL, Elgebaly A, Oishi N, Nakazawa T, Hirayama K, Katoh R, et al. The changing characteristics and molecular profiles of papillary thyroid carcinoma over time: a systematic review. Oncotarget. 2017; 8:10637-10649. https://doi.org/10.18632/oncotarget.12885.

16. Zhao WX, Wang B, Zhang LY, Yan SY, Yang YH. Analysis on the metabolite composition of serum samples from patients with papillary thyroid carcinoma using nuclear magnetic resonance. Int J Clin Exp Med. 2015; 8:18013-18022.

17. Ugurluoglu C, Dobur F, Karabagli P, Celik ZE. Fine needle aspiration biopsy of thyroid nodules: cytologic and histopathologic correlation of 1096 patients. Int J Clin Exp Pathol. 2015; 8:14800-14805.

18. Serrati S, De Summa S, Pilato B, Petriella D, Lacalamita R, Tommasi S, Pinto R. Next-generation sequencing: advances and applications in cancer diagnosis. Onco Targets Ther. 2016; 9:7355-7365.

19. Lan X, Zhang H, Wang Z, Dong W, Sun W, Shao L, Zhang T, Zhang D. Genome-wide analysis of long noncoding RNA expression profile in papillary thyroid carcinoma. Gene. 2015; 569:109-117.
20. Yang M, Tian J, Guo X, Yang Y, Guan R, Qiu M, Li Y, Sun X, Zhen Y, Zhang Y, Chen C, Li Y, Fang H. Long noncoding RNA are aberrantly expressed in human papillary thyroid carcinoma. Oncol Lett. 2016; 12:544-552.

21. Wang Q, Yang H, Wu L, Yao J, Meng X, Jiang H, Xiao C, Wu F. Identification of Specific Long Non-Coding RNA Expression: Profile and Analysis of Association with Clinicopathologic Characteristics and BRAF Mutation in Papillary Thyroid Cancer. Thyroid. 2016; 26:1719-1732.

22. Pan W, Zhou L, Ge M, Zhang B, Yang X, Xiong X, Fu G, Zhang J, Nie X, Li H, Tang X, Wei J, Shao M, et al. Whole exome sequencing identifies lncRNA GAS8-AS1 and LPAR4 as novel papillary thyroid carcinoma driver alternations. Hum Mol Genet. 2016; 25:1875-1884.

23. Liyanarachchi S, Li W, Yan P, Bundschuh R, Brock P, Senter L, Ringel MD, de la Chapelle A, He H. GenomeWide Expression Screening Discloses Long Noncoding RNAs Involved in Thyroid Carcinogenesis. J Clin Endocrinol Metab. 2016; 101:4005-4013.

24. Ma B, Liao T, Wen D, Dong C, Zhou L, Yang S, Wang Y, Ji Q. Long intergenic non-coding RNA 271 is predictive of a poorer prognosis of papillary thyroid cancer. Sci Rep. 2016; 6:36973.

25. Zhang R, Hardin H, Chen J, Guo Z, Lloyd RV. Non-Coding RNAs in Thyroid Cancer. Endocr Pathol. 2016; 27:12-20.

26. Jeong S, Lee J, Kim D, Seol MY, Lee WK, Jeong JJ, Nam KH, Jung SG, Shin DY, Lee EJ, Chung WY, Jo YS. Relationship of Focally Amplified Long Noncoding on Chromosome 1 (FAL1) lncRNA with E2F Transcription Factors in Thyroid Cancer. Medicine (Baltimore). 2016; 95:e2592.

27. Lan X, Sun W, Zhang P, He L, Dong W, Wang Z, Liu S, Zhang H. Downregulation of long noncoding RNA NONHSAT037832 in papillary thyroid carcinoma and its clinical significance. Tumour Biol. 2016; 37:6117-6123.

28. Zhu H, Lv Z, An C, Shi M, Pan W, Zhou L, Yang W, Yang M. Onco-lncRNA HOTAIR and its functional genetic variants in papillary thyroid carcinoma. Sci Rep. 2016; 6:31969.

29. Zhao JJ, Hao S, Wang LL, Hu CY, Zhang S, Guo LJ, Zhang G, Gao B, Jiang Y, Tian WG, Luo DL. Long noncoding RNA ANRIL promotes the invasion and metastasis of thyroid cancer cells through TGF-beta/Smad signaling pathway. Oncotarget. 2016; 7:57903-57918. https:/doi. org/10.18632/oncotarget.11087.

30. Liao T, Qu N, Shi RL, Guo K, Ma B, Cao YM, Xiang J, Lu ZW, Zhu YX, Li DS, Ji QH. BRAF-activated LncRNA functions as a tumor suppressor in papillary thyroid cancer. Oncotarget. 2017; 8:238-247. https://doi.org/10.18632/ oncotarget.10825.

31. Jendrzejewski J, Thomas A, Liyanarachchi S, Eiterman A, Tomsic J, He H, Radomska HS, Li W, Nagy R, Sworczak K, de la Chapelle A. PTCSC3 Is Involved in Papillary Thyroid 
Carcinoma Development by Modulating S100A4 Gene Expression. J Clin Endocrinol Metab. 2015; 100:E1370-1377.

32. Xiang C, Zhang ML, Zhao QZ, Xie QP, Yan HC, Yu X, Wang P, Wang Y. LncRNA-SLC6A9-5:2: A potent sensitizer in 131I-resistant papillary thyroid carcinoma with PARP-1 induction. Oncotarget. 2017; 8:22954-22967. https://doi. org/10.18632/oncotarget.14578.

33. Cha YJ, Koo JS. Next-generation sequencing in thyroid cancer. J Transl Med. 2016; 14:322.

34. Agrawal N, Akbani R, Aksoy BA, Ally A, Arachchi H, Asa SL, Auman JT, Balasundaram M, Balu S, Baylin SB, Behera M, Bernard B, Beroukhim R, et al, and Cancer Genome Atlas Research Network. Integrated genomic characterization of papillary thyroid carcinoma. Cell. 2014; 159:676-690.

35. Santoro M, Melillo RM. Genetics: The genomic landscape of papillary thyroid carcinoma. Nat Rev Endocrinol. 2015; 11:133-134.

36. Killock D. Genetics: The Cancer Genome Atlas maps papillary thyroid cancer. Nat Rev Clin Oncol. 2014; 11:681.

37. Liu B, Chen Y, Yang J. LncRNAs are altered in lung squamous cell carcinoma and lung adenocarcinoma. Oncotarget. 2017; 8:24275-24291. https://doi.org/10.18632/ oncotarget. 13651.

38. Ping Z, Siegal GP, Harada S, Eltoum IE, Youssef M, Shen T, He J, Huang Y, Chen D, Li Y, Bland KI, Chang HR, Shen D. ERBB2 mutation is associated with a worse prognosis in patients with CDH1 altered invasive lobular cancer of the breast. Oncotarget. 2016; 7:80655-80663. https://doi. org/10.18632/oncotarget.13019.

39. Chen Y, Wu JJ, Lin XB, Bao Y, Chen ZH, Zhang CR, Cai Z, Zhou JY, Ding MH, Wu XJ, Sun W, Qian J, Zhang L, et al. Differential lncRNA expression profiles in recurrent gliomas compared with primary gliomas identified by microarray analysis. Int J Clin Exp Med. 2015; 8:5033-5043.

40. Jing SY, Lu YY, Yang JK, Deng WY, Zhou Q, Jiao BH. Expression of long non-coding RNA CRNDE in glioma and its correlation with tumor progression and patient survival. Eur Rev Med Pharmacol Sci. 2016; 20:3992-3996.

41. Zheng J, Liu X, Wang P, Xue Y, Ma J, Qu C, Liu Y. CRNDE Promotes Malignant Progression of Glioma by Attenuating miR-384/PIWIL4/STAT3 Axis. Mol Ther. 2016; 24:1199-1215.

42. Zheng J, Li XD, Wang P, Liu XB, Xue YX, Hu Y, Li Z, Li ZQ, Wang ZH, Liu YH. CRNDE affects the malignant biological characteristics of human glioma stem cells by negatively regulating miR-186. Oncotarget. 2015; 6:25339-25355. https://doi.org/10.18632/oncotarget.4509.

43. Wang Y, Wang Y, Li J, Zhang Y, Yin H, Han B. CRNDE, a long-noncoding RNA, promotes glioma cell growth and invasion through mTOR signaling. Cancer Lett. 2015; 367:122-128.

44. Liu T, Zhang X, Gao S, Jing F, Yang Y, Du L, Zheng G, Li P, Li C, Wang C. Exosomal long noncoding RNA CRNDE-h as a novel serum-based biomarker for diagnosis and prognosis of colorectal cancer. Oncotarget. 2016; 7:85551-85563. https://doi.org/10.18632/oncotarget.13465.

45. Han P, Li JW, Zhang BM, Lv JC, Li YM, Gu XY, Yu ZW, Jia YH, Bai XF, Li L, Liu YL, Cui BB. The lncRNA CRNDE promotes colorectal cancer cell proliferation and chemoresistance via miR-181a-5p-mediated regulation of Wnt/beta-catenin signaling. Mol Cancer. 2017; 16:9.

46. Liu T, Zhang X, Yang YM, Du LT, Wang CX. Increased expression of the long noncoding RNA CRNDE-h indicates a poor prognosis in colorectal cancer, and is positively correlated with IRX5 mRNA expression. Onco Targets Ther. 2016; 9:1437-1448.

47. Song H, Han LM, Gao Q, Sun Y. Long non-coding RNA CRNDE promotes tumor growth in medulloblastoma. Eur Rev Med Pharmacol Sci. 2016; 20:2588-2597.

48. Chen Z, Yu C, Zhan L, Pan Y, Chen L, Sun C. LncRNA CRNDE promotes hepatic carcinoma cell proliferation, migration and invasion by suppressing miR-384. Am J Cancer Res. 2016; 6:2299-2309.

49. Shen S, Liu H, Wang Y, Wang J, Ni X, Ai Z, Pan H, Liu H, Shao Y. Long non-coding RNA CRNDE promotes gallbladder carcinoma carcinogenesis and as a scaffold of DMBT1 and C-IAP1 complexes to activating PI3K-AKT pathway. Oncotarget. 2016; 7:72833-72844. https://doi. org/10.18632/oncotarget.12023.

50. Shao K, Shi T, Yang Y, Wang X, Xu D, Zhou P. Highly expressed lncRNA CRNDE promotes cell proliferation through Wnt/beta-catenin signaling in renal cell carcinoma. Tumour Biol. 2016; 37:15997-6004.

51. Szafron LM, Balcerak A, Grzybowska EA, PienkowskaGrela B, Podgorska A, Zub R, Olbryt M, Pamula-Pilat J, Lisowska KM, Grzybowska E, Rubel T, DansonkaMieszkowska A, Konopka B, et al. The putative oncogene, CRNDE, is a negative prognostic factor in ovarian cancer patients. Oncotarget. 2015; 6:43897-43910. https://doi. org/10.18632/oncotarget.6016.

52. Milewska M, Romano D, Herrero A, Guerriero ML, Birtwistle M, Quehenberger F, Hatzl S, Kholodenko BN, Segatto O, Kolch W, Zebisch A. Mitogen-Inducible Gene-6 Mediates Feedback Inhibition from Mutated BRAF towards the Epidermal Growth Factor Receptor and Thereby Limits Malignant Transformation. PLoS One. 2015; 10:e0129859.

53. Zhang Y, Sui F, Ma J, Ren X, Guan H, Yang Q, Shi J, Ji M, Shi B, Sun Y, Hou P. Positive Feedback Loops Between NrCAM and Major Signaling Pathways Contribute to Thyroid Tumorigenesis. J Clin Endocrinol Metab. 2017; 102:613-624.

54. Robinson MD, Oshlack A. A scaling normalization method for differential expression analysis of RNA-seq data. Genome Biol. 2010; 11:R25.

55. Huang W, Sherman BT, Lempicki RA. Bioinformatics enrichment tools: paths toward the comprehensive functional analysis of large gene lists. Nucleic Acids Res. 2009; 37:1-13. 